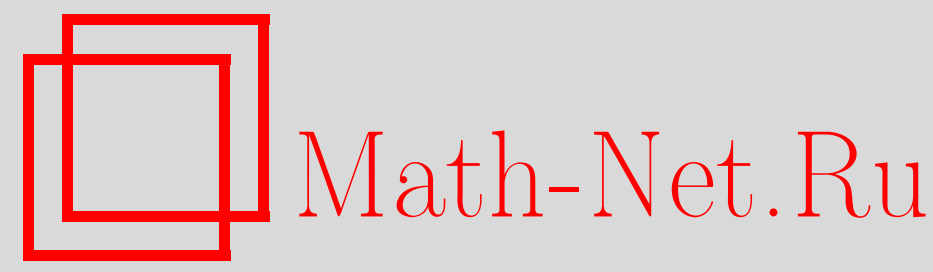

О. И. Богоявленский, Канонические формы инвариантных тензоров и $A$ - $B$ - $C$ когомологии интегрируемых гамильтоновых систем, Матем. сб., 1998, том 189, номер 3, 3-44

DOI: https://doi.org/10.4213/sm302

Использование Общероссийского математического портала Math-Net.Ru подразумевает, что вы прочитали и согласны с пользовательским соглашением

http://www. mathnet.ru/rus/agreement

Параметры загрузки:

IP: 54.162 .27 .143

26 апреля 2023 г., $14: 13: 01$

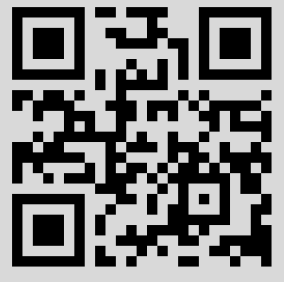


УДК 517.9

\title{
О.И. Богоявленский
}

\section{Канонические формы инвариантных тензоров и $A$ - $B$ - $C$-когомологии интегрируемых гамильтоновых систем}

\begin{abstract}
Выведены канонические формы для тензоров типа $(\ell, m)$ при $\ell+m \leqslant 3$, которые являются инвариантными по отношению к некоторой интегрируемой по Лиувиллю невырожденной гамильтоновой системе $V$ на симплектическом многообразии $M^{2 k}$. Доказано, что характеристический многочлен любого инвариантного тензора $A_{\beta}^{\alpha}$ типа $(1,1)$ является полньп квадратом; поэтому его собственные значения имеют четные кратности. Любая инвариантная метрика $g_{\alpha \beta}$ является неопределенной и имеет сигнатуру $\sigma \leqslant k$. Выведенные канонические формы применены для вычисления $A-B$ - $C$-когомологий интегрируемых по Лиувиллю гамильтоновых систем.

Библиография: 22 названия
\end{abstract}

\section{§1. Введение}

I. Данная работа продолжает исследования тензорных инвариантов динамических систем, которые были начаты в статьях [1]-[5]. Основные применения тензорных инвариантов связаны с гамильтоновьми системами

$$
\dot{x}^{\alpha}=V^{\alpha}(x)=P_{1}^{\alpha \beta} H_{, \beta}, \quad H_{, \beta}=\partial H / \partial x^{\beta}
$$

на пуассоновых многообразиях $M^{2 k}$ с невырожденной пуассоновой структурой $P_{1}^{\alpha \beta}$.

ОПРЕДЕЛЕНИЕ 1.1. Гамильтонова система (1.1) называется $C$-интегрируемой, если она является интегрируемой по Лиувиллю [6], [7] и все инвариантные подмногообразия постоянного уровня ее $k$ инволютивных и функционально независимых первых интегралов компактны.

Класс $C$-интегрируемых гамильтоновых систем особенно важен, потому что он лежит в основании теории Колмогорова-Арнольда-Мозера [8]-[12], которая изучает малые гамильтоновы возмушения этих систем. Тензорные инварианты этих систем доставляют инвариантную интерпретацию известных достаточных условий применимости теории КАМ. Теорема Колмогорова [8]-[10] утверждает, что условие невырожденности $C$-интегрируемой системы (1.1) и условие ее изоэнергетической невырожденности являются достаточньми условиями для применимости теории КАМ к малым возмущениям этой системы. Оба условия невырожденности были введены Пуанкаре в 1892 году [13]. Теория тензорных инвариантов, развитая в работах $[3],[5]$, дает симметрийную интерпретацию этих двух условий: 
ДВА ДОСТАТОЧНЫХ УСЛОВИЯ ДЛЯ ПРИМЕНИМОСТИ ТЕОРИИ КАМ.

1) Алгебра Ли симметрий $C$-интегрируемой гамильтоновой системь $V$ является абелевой.

2) Алгебра Ли изоэнергетических конформных симметрий системы $V$ является абелевой.

В работе [5] мы получили следующее

НЕОБХоДИМОе УСЛОВИЕ ДЛЯ ПРИМЕНИМОСТИ теОРИИ КАМ. Алгебра ЛиПуассона первых интегралов $C$-интегрируемой гамильтоновой системъ $V$ обязана быть абелевой.

Теория тензорных инвариантов имеет другое, неожиданное, применение к теории КАМ, которое заключается в конструкции [3] широкого класса негамильтоновых возмушений, динамика которых эквивалентна динамике малых гамильтоновых возмушений в теории КАМ.

Теория тензорных инвариантов привела нас к определению $A-B-C$-когомологий динамических систем, которые мы изучали в работах [2], [4], [5]. В статье [5] были введены $B$-когомологии диффеоморфизмов гладких многообразий. В $\S \S 13,14$ данной работы мы вычисляем $A-B$ - $C$-когомологии малых размерностей для $C$-интегрируемых гамильтоновых систем и некоторых диффеоморфизмов $f: M^{2 k} \rightarrow M^{2 k}$.

Имеется некоторая параллель между теорией тензорных инвариантов интегрируемых динамических систем и теорией алгебраических инвариантов, созданной Гильбертом в 1890-1893 годах. Обе теории дают новую перспективу, исследуя основные инвариантные объекты, связанные с базисными симметриями. Однако, в то время как теория Гильберта не имела первоначально применений вне контекста, в котором она была создана, теория тензорных инвариантов интегрируемых динамических систем определенно имеет применения в конкретных задачах динамики, математической физики и механики. Некоторые из этих применений указаны выше и подробно исследованы в работах [3], [5].

ОПреДЕЛЕниЕ 1.2 . Тензор $T_{m}^{\ell}$ типа $(\ell, m)$ на многообразии $M^{2 k}$ называется $C$-инвариантныцм, если он инвариантен по отношению к $C$-интегрируемой гамильтоновой системе (1.1), которая является невырожденной в смысле Пуанкаре.

В данной работе мы изучаем $C$-инвариантные тензоры $T_{m}^{\ell}$ при $\ell+m \leqslant 3$. Именно эти тензоры играют наиболее важную роль в теории интегрируемых гамильтоновых систем. $C$-инвариантные тензоры $U^{\alpha}$ типа $(1,0)$ соответствуют симметриям этих систем. Тензоры $\theta_{\alpha}$ типа $(0,1)$ являются дифференциальными 1-формами. Тензоры $h_{\alpha \beta}$ типа $(0,2)$ появляются как инвариантные симплектические структуры $\omega_{\alpha \beta}$ и инвариантные метрики $g_{\alpha \beta}$. Тензоры типа $(2,0)$ имеют применения как пуассоновы структуры $P^{\alpha \beta}$. $C$-инвариантные тензоры $A_{\beta}^{\alpha}$ типа $(1,1)$ появляются в виде рекурсивњ операторов в теории бигамильтоновых систем. $C$-инвариантные тензоры $N_{\beta \gamma}^{\alpha}$ типа $(1,2)$ появляются как тензоры Нейенхейса [14] рекурсивных операторов $A_{\beta}^{\alpha}$. Тензоры $T_{\alpha \beta \gamma}$ типа $(0,3)$ имеют применения как $C$-инвариантные дифференциальные 3 -формы. $C$-инвариантные тензоры $R^{\alpha \beta \gamma}$ типа $(3,0)$ возникают как скобки Схоутена двух общих пуассоновых структур для $C$-интегрируемых гамильтоновых систем. Тензоры $S_{\gamma}^{\alpha \beta}$ типа $(2,1)$ описьвают инвариантные алгебраические структуры в кокасательном расслоении $T^{*}\left(M^{n}\right)$.

II. Результаты статей [1]-[5] приводят к следующей общей проблеме. 
ПРОБЛЕМА КАНОНИЧЕСКИХ ФОРМ. Какой вид имеют канонические формъ C-инвариантных тензоров $T_{m}^{\ell}$ типа $(\ell, m)$ ?

В серии предложений, мы выводим канонические формы тензоров $T_{m}^{\ell}$ при $\ell+m \leqslant 3$, которые инвариантны по отношению к $C$-интегрируемой невырожденной по Пуанкаре гамильтоновой системе $V$ :

$$
\dot{I}_{j}=0, \quad \dot{\varphi}_{j}=\frac{\partial H(I)}{\partial I_{j}}, \quad j=1, \ldots, k
$$

в переменных действие-угол $I_{j}, \varphi_{j}$. Переменные действия $I_{j}$ изменяются в шаре $B_{a} \in \mathbb{R}^{k}$, угловые переменные $\varphi_{j}$ пробегают тор $\mathbb{T}^{k}$. Канонические формы $C$-инвариантных тензоров $T_{m}^{\ell}$ типа $(\ell, m)$ выведены с помошью следуюшего метода.

a) Уравнение инвариантности $L_{V} T_{m}^{\ell}=0$ сводится к системе линейных обыкновенных дифференциальных уравнений в пространстве тензорных компонент $T_{m}^{\ell}$ (всюду $L_{V}$ означает производную Ли по отношению к векторному полю $V$ ).

б) Возникающая линейная динамическая система преобразуется к верхне-треугольному виду с постоянньми коэффициентами в некоторой специальной системе координат.

в) На основе обнаруженной верхне-треугольной структуры полученная линейная динамическая система интегрируется в явном виде. Выведенные формулы показывают, что область значений компонент $C$-инвариантных тензоров $T_{m}^{\ell}$ является линейным подпространством, которое состоит из особых точек этой динамической системы. Именно это особое множество и определяет канонические формы $C$-инвариантных тензоров $T_{m}^{\ell}$.

Этот метод является общим и применим к исследованию $C$-инвариантных тензоров $T_{m}^{\ell}$ произвольных валентностей $\ell$ и $m$.

III. Основные результаты данной работы сформулированы в инвариантном виде в следуюшей теореме. Всюду $L_{U}$ означает производную Ли по отношению $\mathrm{k}$ произвольной симметрии $U$, и $i_{U}$ означает оператор внутреннего умножения [15].

Теорема 1. Предположим, ито тензор $T_{m}^{\ell}$ типа $(\ell, m)$ на пуассоновом многообразии $M^{2 k}$ инвариантен по отношению $к$ С-интегрируемой невырожденной гамильтоновой системе $V$. Тогда следующие свойства справедливы для любых симметрий $U$ и $U_{1}$ системы $V$ и любых $C$-инвариантных 1-форм $\theta$ и $\theta_{1}$.

1) Любая С-инвариантная дифференциальная 1-форма $\theta$ удовлетворяет уравнениям

$$
\operatorname{rank} d \theta \leqslant k, \quad \theta(U)=0, \quad L_{U} \theta=0, \quad i_{U} d \theta=0 .
$$

2) При $\ell+m=2$ справедливо уравнение

$$
\operatorname{rank}\left\|L_{U} T_{m}^{\ell}\right\| \leqslant k
$$

3) Характеристический многочлен $P(\lambda, x)=\operatorname{det}(A(x)-\lambda)$ любого $C$-инвариантного тензора $A(x)$ типа $(1,1)$ является полным квадратом, $P(\lambda, x)=$ $Q^{2}(\lambda, x)$. Поэтому собственные значения любого $C$-инвариантного тензора $A(x)$ типа $(1,1)$ имеют четные кратности. 
4) Любой $C$-инвариантный тензор $h_{\alpha \beta}$ типа $(0,2)$ и любой $C$-инвариантный тензор $P^{\alpha \beta}$ типа $(2,0)$ удовлетворяют уравнениям $h\left(U, U_{1}\right)=0, P\left(\theta, \theta_{1}\right)=0$. Любая С-инвариантная метрика $g_{\alpha \beta}=g_{\beta \alpha}$ является неопределенной $c$ сигнатурой $\sigma=\left|n_{+}-n_{-}\right| \leqslant k$. Числа $n_{+} u n_{-}$ее положсительных $и$ отрицательных квадратов удовлетворяют неравенствам $n_{+} \leqslant k, n_{-} \leqslant k$. При $n=2 k=4 \ell+2$ любая $C$-инвариантная метрика $g_{\alpha \beta}$ является вырожденной.

5) При $\ell+m \leqslant 3$ любой $C$-инвариантный тензор $T_{m}^{\ell}$ типа $(\ell, m)$ удовлетворяет уравнению

$$
L_{U_{1}} L_{U} T_{m}^{\ell}=0
$$

6) Любой $C$-инвариантный тензор $N_{\beta \gamma}^{\alpha}$ типа $(1,2)$ и любой $C$-инвариантный тензор $S_{\gamma}^{\alpha \beta}$ типа $(2,1)$ удовлетворяют соотношениям

$$
\begin{array}{ll}
\operatorname{rank}\left\|N_{\beta \tau}^{\alpha} U^{\tau}\right\| \leqslant k, & \operatorname{rank}\left\|N_{\beta \gamma}^{\tau} \theta_{\tau}\right\| \leqslant k, \\
\operatorname{rank}\left\|S_{\tau}^{\alpha \beta} U^{\tau}\right\| \leqslant k, & \operatorname{rank}\left\|S_{\beta}^{\alpha \tau} \theta_{\tau}\right\| \leqslant k .
\end{array}
$$

Любой $C$-инвариантный тензор $T_{\alpha \beta \gamma}$ типа $(0,3)$ и любой $C$-инвариантный тензор $R^{\alpha \beta \gamma}$ типа $(3,0)$ удовлетворяют соотношениям

$$
\operatorname{rank}\left\|T_{\alpha \beta \tau} U^{\tau}\right\| \leqslant k, \quad \operatorname{rank}\left\|R^{\alpha \beta \tau} \theta_{\tau}\right\| \leqslant k
$$

7) При $\ell+m=3$ все возможнне свертки $C$-инвариантных тензоров $N_{\beta \gamma}^{\alpha}, S_{\gamma}^{\alpha \beta}, T_{\alpha \beta \gamma} u R^{\alpha \beta \gamma}$ с двумя произвольными симметриями или двумя произвольными С-инвариантными 1-формами равны нулю.

Доказательство теоремы 1 разбито на девять частей, которые представлены в $\S \S 3-12$.

Свойства $C$-инвариантных тензоров $T_{m}^{l}$, указанные в теореме 1 , имеют применения в качестве необходимых условий для невырожденной $C$-интегрируемости данной динамической системы $V$.

IV. Канонические формы $C$-инвариантных тензоров и теорема 1 применяются в $\S \S 13$ и 14 для вычисления $A-B$ - $C$-когомологий $C$-интегрируемых невырожденных гамильтоновых систем. Мы доказываем, что первые пять $B$-когомологий таких динамических систем $V$ в инвариантных тороидальных областях $\mathscr{O}=B_{a} \times \mathbb{T}^{k}$ имеют вид

$$
\begin{gathered}
H_{B}^{0}(V, \mathscr{O})=\mathbb{R}^{1}, \quad H_{B}^{1}(V, \mathscr{O})=0, \quad H_{B}^{2}(V, \mathscr{O})=\mathbb{R}^{\infty}, \\
H_{B}^{3}(V, \mathscr{O})=0, \quad H_{B}^{4}(V, \mathscr{O})=\mathbb{R}^{\infty} .
\end{gathered}
$$

Поэтому бесконечномерность четных когомологий $H_{B}^{2}\left(V, M^{2 k}\right)$ и $H_{B}^{4}\left(V, M^{2 k}\right)$ является необходимым условием для невырожденной $C$-интегрируемости данной гамильтоновой системы $V$. 


\section{§2. Предварительные результаты}

I. Напомним, что гамильтонова система (1.1) назьвается полностью интегрируемой по Лиувиллю [6], [7], [16], если существуют $k=n / 2$ инволютивных функционально-независимых первых интегралов $F_{1}(x), \ldots, F_{k}(x)$ этой системы:

$$
\left\{F_{j}, F_{\ell}\right\}=P_{1}^{\alpha \beta} F_{j, \alpha} F_{\ell, \beta}=0 .
$$

В данной статье все греческие индексы $\alpha, \beta, \gamma, \delta$ изменяются между 1 и $n=2 k$. Все латинские индексы $i, j, \ell, m$ изменяются между 1 и $n=k$. Всюду подразумевается суммирование по повторяюшимся индексам.

Классическая теорема Лиувилля [6], [7] утверждает, что почти все многообразие $M^{n}$ (исключая некоторое инвариантное подмножество $S \subset M^{n}, \operatorname{dim} S \leqslant n-1$ ) покрыто системой открытых тороидальных областей $\mathscr{O}_{m} \subset M^{n}$ с координатами действие-угол $I_{1}, \ldots, I_{k}, \varphi_{1}, \ldots, \varphi_{k}$. В этих координатах интегрируемая по Лиувиллю система (1.1) имеет вид

$$
\dot{I}_{j}=0, \quad \dot{\varphi}_{j}=\frac{\partial H}{\partial I_{j}}, \quad H=H\left(I_{1}, \ldots, I_{k}\right) .
$$

Симплектическая структура $\omega_{1}=P_{1}^{-1}$ имеет канонический вид

$$
\omega_{1}=d I_{j} \wedge d \varphi_{j}, \quad \omega_{1}=P_{1}^{-1} .
$$

Координаты действия $I_{1}, \ldots, I_{k}$ определены в шаре

$$
B_{a}: \sum_{j=1}^{k}\left(I_{j}-I_{j 0}\right)^{2}<a^{2} .
$$

Угловые координаты $\varphi_{1}, \ldots, \varphi_{k}$ пробегают тор $\mathbb{T}^{k}, 0 \leqslant \varphi_{j} \leqslant 2 \pi$, в компактном случае и торический цилиндр $\mathbb{T}^{m} \times \mathbb{R}^{k-m}, 0 \leqslant m<k$, если многообразие $I_{j}(x)=$ $I_{j 0}$ является некомпактньм.

Полностью интегрируемая гамильтонова система (1.1), (2.1) называется невырожденной, если условие Пуанкаре [13] для гессиана

$$
\operatorname{det}\left\|\frac{\partial^{2} H(I)}{\partial I_{\alpha} \partial I_{\beta}}\right\| \neq 0
$$

вьполнено в открытой плотной области в переменных действие-угол

$$
I_{1}, \ldots, I_{k}, \quad \varphi_{1}, \ldots, \varphi_{k}, \quad \varphi_{i}=\varphi_{i} \bmod (2 \pi) .
$$

Инвариантные подмногообразия $C$-интегрируемой гамильтоновой системы (1.1) являются торами $\mathbb{T}^{k}$ :

$$
\mathbb{T}^{k}: \quad I_{1}=c_{1}, \ldots, I_{k}=c_{k}, \quad 0 \leqslant \varphi_{i} \leqslant 2 \pi .
$$

Соответствуюшие касательные подпространства определяют $k$-мерное распределение

$$
\mathscr{L}_{x}=T_{x}\left(\mathbb{T}^{k}\right) \subset T_{x}\left(M^{n}\right) .
$$


II. Условие невырожденности по Пуанкаре (2.3) означает, что $k$ функций

$$
J_{\ell}(I)=\frac{\partial H(I)}{\partial I_{\ell}}
$$

образуют систему локальных координат в шаре $B_{a}(2.2)$. Гамильтонова система (2.1) принимает вид

$$
\dot{J}_{i}=0, \quad \dot{\varphi}_{i}=J_{i}
$$

в локальных координатах

$$
J_{1}, \ldots, J_{k}, \quad \varphi_{1}, \ldots, \varphi_{k}, \quad \varphi_{i}=\varphi_{i} \bmod (2 \pi) .
$$

Преимущество использования неканонических координат $J_{i}, \varphi_{i}$ состоит в том, что гамильтонова система $(1.1),(2.1)$ принимает наиболее простой вид (2.6) в координатах (2.7). Наши результаты относительно алгебраических свойств инвариантных тензоров доказаны в тороидальных областях $\mathscr{O}_{m}$, которые покрывают все многообразие $M^{n}$ за исключением некоторого инвариантного подмножества $S$, $\operatorname{dim} S \leqslant n-1$. Гладкость рассматриваемых $C$-инвариантных тензоров влечет непрерывное продолжение их алгебраических свойств на все многообразие $M^{n}$, независимо от выбора локальных координат.

Произвольная траектория динамической системы (2.6) является всюду плотной на торе $\mathbb{T}^{k}(2.4)$, если и только если соответствующие $k$ координат $J_{1}, \ldots, J_{k}$ рационально независимы. Это означает, что для произвольных целых чисел $m_{1}, \ldots, m_{k},\left(m_{1}, \ldots, m_{k}\right) \neq(0, \ldots, 0)$, справедливо неравенство

$$
m_{1} J_{1}+\cdots+m_{k} J_{k} \neq 0
$$

Это условие справедливо почти всюду в шаре $B_{a}(2.2)$. Поэтому траектории $C$-интегрируемой невырожденной гамильтоновой системы $(2.1),(2.6)$ являются всюду плотньми на почти всех торах (2.4). Следовательно, любой гладкий первый интеграл $F\left(J_{i}, \varphi_{i}\right)$ системы $(2.6)$ является постоянным на всех торах $\mathbb{T}^{k}$ и поэтому зависит только от переменных действия

$$
\frac{d F}{d t}=0 \quad \Longrightarrow \quad F=F\left(J_{1}, \ldots, J_{k}\right)
$$

III. Координаты (2.7) определяют базис касательных векторов

$$
e_{i}=\frac{\partial}{\partial J_{i}}, \quad e_{i+k}=\frac{\partial}{\partial \varphi_{i}}
$$

в касательном пространстве $T_{x}\left(M^{n}\right)$ и двойственный базис

$$
e^{i}=d J_{i}, \quad e^{i+k}=d \varphi_{i}
$$

в кокасательном пространстве $T_{x}^{*}\left(M^{n}\right)$. Касательные векторы обозначаются $u, v, w$, кокасательные векторы обозначаются $u^{*}, v^{*}, w^{*}$. Внутреннее произведение определяется формулой

$$
\left\langle u, u^{*}\right\rangle=u^{*}(u)
$$

Два базиса (2.9) и (2.10) связаны соотношениями $\left\langle e_{i}, e^{j}\right\rangle=\delta_{i}^{j}$. 
В любой инвариантной тороидальной области $\mathscr{O}$ мы вводим нильпотентный оператор $D$. Этот оператор действует на касательном пространстве $T_{x}\left(M^{n}\right)$ по формулам

$$
D e_{i}=e_{i+k}, \quad D e_{i+k}=0, \quad D^{2}=0 .
$$

Оператор $D$ действует на кокасательном пространстве $T_{x}^{*}\left(M^{n}\right)$ по формулам

$$
D e^{i}=0, \quad D e^{i+k}=e^{i} .
$$

Очевидно справедливо тождество $\left\langle D u, u^{*}\right\rangle=\left\langle u, D u^{*}\right\rangle$ для любого вектора $u \in T_{x}\left(M^{n}\right)$ и любого ковектора $u^{*} \in T_{x}\left(M^{n}\right)$.

Оператор $D(x)$ порождает некоторое дифференцирование [15] тензорной алгебры $T\left(T\left(M^{n}\right)\right)$. Это дифференцирование также обозначается $D(x)$.

IV. Векторное поле $U$ на многообразии $M^{n}$ называется симметрией динамической системы $\dot{x}^{i}=V^{i}(x)$, если оно коммутирует с векторньм полем $V$ :

$$
[U, V]=0 .
$$

В этом случае векторное поле $U$ является инвариантным по отношению к динамической системе $\dot{x}^{i}=V^{i}(x)$.

В работе [5] изучались симметрии обших динамических систем с квазипериодической динамикой

$$
\dot{I}_{1}=0, \ldots, \dot{I}_{p}=0, \quad \dot{\varphi}_{1}=\omega_{1}(I), \ldots, \dot{\varphi}_{q}=\omega_{q}(I)
$$

в тороидальных областях $\mathscr{O}=B_{a} \times \mathbb{T}^{q} \subset M^{n}$ при произвольных размерностях $p$ и $q, p+q=n$. Пусть $X \subset B_{a}$ - множество точек $I \in B_{a}$, для которых траектории системы (2.14) являются всюду плотными на торах $T^{q}$.

ОПРеДЕЛЕнИЕ 2.1. Динамическая система (2.14) называется $T^{q}$-плотной в торической области $\mathscr{O}=B_{a} \times \mathbb{T}^{q} \subset M^{n}$, если множество $X$ является всюду плотньм в шаре $B_{a}$.

В работе [5] мы доказали следуюшую лемму.

Лемма 2.1. Векторное поле $U$ тогда и только тогда является симметрией $\mathbb{T}^{q}$-плотной динамической системы (2.14), если оно имеет вид

$$
U=\sum_{j=1}^{p} U^{j}(I) \frac{\partial}{\partial I_{j}}+\sum_{\alpha=1}^{q} U^{\alpha+p}(I) \frac{\partial}{\partial \varphi_{\alpha}} .
$$

Здесь гладкие функции $U^{\alpha+p}(I)$ являются произвольньми и гладкие функиии $U^{j}(I)$ удовлетворяют системе линейных уравнений

$$
\sum_{j=1}^{p} \frac{\partial \omega_{\alpha}(I)}{\partial I_{j}} U^{j}(I)=0, \quad \alpha=1, \ldots, q .
$$

Применяя эту лемму к динамической системе (2.6), мы получаем, что любая ее симметрия $U$ имеет вид

$$
U=\sum_{i=1}^{k} U^{i+k}(J) \frac{\partial}{\partial \varphi_{i}},
$$

где $U^{i+k}(J)$ являются произвольными гладкими функциями.

Формула (2.15) приводит к выражению для частных производных

$$
U_{, \beta}^{\alpha}=\delta_{i+p}^{\alpha} \delta_{\beta}^{\ell} U_{, \ell}^{i+p}(J),
$$

которое используется в дальнейшем. 
СЛЕДСТВИЕ 2.1. Любая симметрия $U$ С-интегрируемой невырождденной гамильтоновой системы (1.1) удовлетворяет уравнению $D U=0$. Векторное поле $U$ касается $k$-мерного распределения $\mathscr{L}_{x}(2.5)$. Соответствующая динамическая система

$$
\dot{x}^{\alpha}=U^{\alpha}\left(x^{1}, \ldots, x^{n}\right)
$$

является интегрируемой. Первые интеграль $F(x)$ системы (1.1) одновременно являются первыми интегралами системы (2.17).

Действительно, ввиду (2.15), система (2.17) имеет вид

$$
\dot{J}_{i}=0, \quad \dot{\varphi}_{i}=U^{i+k}(J)
$$

в тороидальных координатах (2.7). Следствие 2.1 легко выводится из формулы (2.18).

\section{§ 3. $C$-Инвариантные дифференциальные 1-формы}

І. Любая дифференциальная 1-форма $\theta$ имеет вид

$$
\theta=\theta_{i}(J, \varphi) d J_{i}+\theta_{i+k}(J, \varphi) d \varphi_{i}
$$

в тороидальных координатах (2.7).

ПРЕДЛОЖЕНИЕ 3.1. Дифференциальная 1-форма $\theta$ является интегрируемой по отночению $к$ С-инвариантной невырожденной гамильтоновой системе (1.1) тогда и только тогда, если она имеет вид

$$
\theta=\theta_{i}(J) d J_{i}
$$

в координатах $J_{i}, \varphi_{i}(2.7)$.

ДокАЗАтЕльство. Векторное поле $V$, определенное динамической системой (2.6), имеет компоненты

$$
V^{i}=0, \quad V^{i+k}=J_{i} .
$$

Эти формулы влекут

$$
V_{, \beta}^{\alpha}=\delta_{i+k}^{\alpha} \delta_{\beta}^{i}
$$

где подразумевается суммирование по отношению к индексу $i=1, \ldots, k$. Уравнение инвариантности $L_{V} \theta=0$ для дифференциальной 1-формы $\theta$ имеет вид

$$
\left(L_{V} \theta\right)_{\beta}=\dot{\theta}_{\beta}+V_{, \beta}^{\alpha} \theta_{\alpha}=0 .
$$

Здесь оператор $L_{V}$ означает производную Ли по отношению к векторному полю $V$. После подстановки формул (3.1) и (3.4), уравнение (3.5) дает

$$
\dot{\theta}_{i}=-\theta_{i+k}, \quad \dot{\theta}_{i+k}=0 .
$$

Вследствие (2.8), решения уравнений (3.6) имеют вид

$$
\theta_{i}(t)=-\widetilde{\theta}_{i+k}(J) t+\widetilde{\theta}_{i}(J), \quad \theta_{i+k}(t)=\widetilde{\theta}_{i+k}(J),
$$

где $\widetilde{\theta}_{i}(J)$ и $\widetilde{\theta}_{i+k}(J)$ являются произвольными гладкими функциями. Компоненты $\theta_{\alpha}(J, \varphi)$ любой гладкой дифференциальной 1-формы (3.1) ограничены по модулю на любом торе $\mathbb{T}^{k}(2.4)$. Решения (3.7) являются ограниченными при любом $t$ тогда и только тогда, если $\widetilde{\theta}_{i+k}(J)=0$ при $i=1, \ldots, k$. Поэтому, используя $(3.7)$ и тот факт, что общие траектории $C$-интегрируемой невырожденной гамильтоновой системы (1.1), (2.6) всюду плотны на торах $\mathbb{T}^{k}$, мы получаем, что 1-форма $\theta$ является $C$-инвариантной тогда и только тогда, если она имеет вид $(3.2)$.

Лемма Пуанкаре и предложение 3.1 приводят к следствию: 
Любая замкнутая $C$-инвариантная 1-форма $\theta$ является точной в любой тороидальной области $\mathscr{O}=B_{a} \times \mathbb{T}^{k}$.

II. ТЕОРемА 1, чАСТь 1. Если динамическая система $V$

$$
\dot{x}^{i}=V^{i}\left(x^{1}, \ldots, x^{n}\right)
$$

на гладком многообразии $M^{2 k}$ является $C$-интегрируемой невырожденной гамильтоновой системой, то выполняются следующие необходимые условия.

1) Любая С-инвариантная дифференциальная 1-форма $\theta$ удовлетворяет уравнениям

$$
\theta(V)=0, \quad i_{V} d \theta=0, \quad \operatorname{rank} d \theta \leqslant k .
$$

При $k=2 l$ справедливь уравнения

$$
\theta \wedge d \theta \wedge \cdots \wedge d \theta=0
$$

где число сомножстелей равно $\left[\frac{n}{4}\right]+1=\ell+1$. При $k=2 \ell+1$ справедливь уравнения

$$
d \theta \wedge \cdots \wedge d \theta=0
$$

где число сомнохителей равно $\left[\frac{n}{4}\right]+1=\ell+1$.

2) Любая симметрия $U$ и любая С-инвариантная дифференциальная 1-форма $\theta$ удовлетворяют уравнениям

$$
\theta(U)=0, \quad i_{U} d \theta=0, \quad L_{U} \theta=0
$$

ДокАЗАТЕЛьство. 1) Применяя предложение 1, мы получаем, что если динамическая система (3.8) является $C$-интегрируемой, то любая инвариантная 1-форма $\theta$ имеет вид (3.2) в тороидальных координатах (2.7). Отсюда следует (3.9). Внешние произведения (3.10) и (3.11) имеют степень $k+1$. Поэтому для 1-фороы $\theta(3.2)$, которая зависит только от $k$ переменных $J_{1}, \ldots, J_{k}$, мы получаем

$$
\theta \wedge d \theta \wedge \cdots \wedge d \theta=0, \quad d \theta \wedge \cdots \wedge d \theta=0
$$

при $k=2 \ell$ и $k=2 \ell+1$ соответственно.

2) Применяя лемму 2.1 и предложение 3.1, мы получаем, что если динамическая система (3.8) является $C$-интегрируемой, то любая симметрия $U$ имеет вид (2.15) и любая $C$-инвариантная 1 -форма $\theta$ имеет вид $(3.2)$ в координатах $(2.7)$. Отсюда мы находим

$$
\theta(U)=0, \quad d \theta(U, W)=0
$$

для любого касательного вектора $W$. Второе уравнение (3.13) означает, что $i_{U} d \theta=0$. Поэтому, применяя $(3.13)$ и формулу Картана $[15],[17],[18]$

$$
L_{U}=i_{U} \circ d+d \circ i_{U}
$$

мы получаем

$$
L_{U} \theta=i_{U} d \theta+d(\theta(U))=0 .
$$

Следовательно, любая симметрия $U$ системы (3.8) сохраняет любую $C$-инвариантную 1-форору $\theta$. 
Уравнения (3.9)-(3.11) не являются независимыми. Действительно, формула Картана (3.14) влечет

$$
L_{V} \theta=i_{V} d \theta+d(\theta(V))=0
$$

для инвариантной 1-формы $\theta$. Ввиду (3.15) равенство $\theta(V)=0$ влечет $i_{V} d \theta=0$. Стандартные методы линейной алгебры доказывают, что (3.10) и (3.11) влекут неравенство rank $d \theta \leqslant k$.

III. Пусть $M^{2 k}$ - симплектическое многообразие с симплектической структурой $\omega$. Предположим, что $\omega=d \theta$ в некоторой области $\mathscr{D} \subset M^{2 k}$.

СлЕДСТВИЕ 3.1. Если некоторая гамильтонова система

$$
\dot{x}^{\alpha}=V^{\alpha}=\left(\omega^{-1}\right)^{\alpha \beta} H_{, \beta}
$$

в инвариантной области $\mathscr{D}$ сохраняет 1-форму $\theta, d \theta=\omega$, то эта система не является $C$-интегрируемой и невырожденной в любой инвариантной подобласти $\mathscr{O}_{a} \subset \mathscr{D}$.

ДокАЗАТЕЛЬСтво. Вследствие $d \theta=\omega$, инвариантная 1-форма $\theta$ удовлетворяет уравнениям

$$
i_{V} d \theta=-d H, \quad \operatorname{rank} d \theta=2 k .
$$

Эти уравнения не совместимы с необходимыми условиями (3.9).

Следствие 3.1 имеет применение к гамильтоновым системам

$$
\dot{p}_{i}=-\frac{\partial H}{\partial q_{i}}, \quad \dot{q}_{i}=\frac{\partial H}{\partial p_{i}}
$$

с однородньми степени 2 гамильтоновьми функциями $H(p, q)$. Эти гамильтоновы системы сохраняют 1-форму $\theta=p_{i} d q_{i}-q_{i} d p_{i}$, которая удовлетворяет уравнению $d \theta=2 \omega$. Действительно, дифференцируя 1-форму $\theta$ по отношению к системе (3.16) и применяя теорему Эйлера об однородных функциях, мы получаем

$$
\dot{\theta}=d\left(p_{i} \frac{\partial H}{\partial p_{i}}+q_{i} \frac{\partial H}{\partial q_{i}}-2 H\right)=0
$$

поскольку гамильтониан $H(p, q)$ является однородной функцией степени 2. Применяя следствие 3.1 , мы получаем, что любая гамильтонова система (3.16) с однородной степени 2 гамильтоновой функцией $H(p, q)$ или не является $C$-интегрируемой, или вырождена.

СлЕДСТвИЕ 3.2. Любая С-инвариантная 1-форма $\theta$ удовлетворяет уравнению $D \theta=0$. С-инвариантные дифференциальные формы $\theta$ и $d \theta$ аннулируют $k$-мерное распределение $\mathscr{L}_{x}(2.5)$ :

$$
\theta\left(\mathscr{L}_{x}\right)=0, \quad d \theta\left(\mathscr{L}_{x}, T_{x}\left(M^{n}\right)\right)=0 .
$$

Доказательство следует из формул (3.2) и определения оператора дифференцирования $D(2.13)$. 


\section{$\S 4$. Общие $C$-инвариантные тензоры типа $(0,2)$}

I. Представим тензор $h_{\alpha \beta}(J, \varphi)$ типа $(0,2)$ в блочном виде

$$
h_{\alpha \beta}(J, \varphi)=\left(\begin{array}{ll}
b_{1} & b_{3} \\
b_{2} & b_{4}
\end{array}\right)
$$

в тороидальных координатах $J_{i}, \varphi_{i}(2.7)$. Здесь $b_{1}, b_{2}, b_{3}, b_{4}$ - матрицы размера $k \times k$.

ПРЕДЛОЖЕНИЕ 4.1. Тензор $h_{\alpha \beta}$ типа $(0,2)$ является инвариантныц по отношению $К$ С-интегрируемой невырожденной гамильтоновой системе (1.1) тогда и только тогда, если он имеет блочный вид

$$
h_{\alpha \beta}=\left(\begin{array}{cc}
a(J) & b(J) \\
-b(J) & 0
\end{array}\right)
$$

в координатах (2.7). Здесь $а(J)$ и $b(J)$ - произвольнье $k \times k$ матрицы.

ДокАЗАТЕЛЬСтво. Уравнение инвариантности для тензора $h$ типа $(0,2)$ имеет вид

$$
\left(L_{V} h\right)_{\alpha \beta}=\dot{h}_{\alpha \beta}+V_{, \alpha}^{\gamma} h_{\gamma \beta}+V_{, \beta}^{\gamma} h_{\alpha \gamma}=0 .
$$

Вследствие (3.4) и (4.1), система (4.3) эквивалентна системе четырех линейных матричных уравнений

$$
\dot{b}_{1}=-b_{2}-b_{3}, \quad \dot{b}_{2}=-b_{4}, \quad \dot{b}_{3}=-b_{4}, \quad \dot{b}_{4}=0 .
$$

Эта система очевидно имеет верхне-треугольную структуру. Применяя (2.8), мы получаем, что все решения уравнений (4.4) имеют вид

$$
\begin{gathered}
b_{1}(t)=\widetilde{b}_{4}(J) t^{2}-\left(\widetilde{b}_{2}(J)+\widetilde{b}_{3}(J)\right) t+\widetilde{b}_{1}(J) \\
b_{2}(t)=-\widetilde{b}_{4}(J) t+\widetilde{b}_{2}(J), \quad b_{3}(t)=-\widetilde{b}_{4}(J) t+\widetilde{b}_{3}(J), \quad b_{4}(t)=\widetilde{b}_{4}(J) .
\end{gathered}
$$

Компоненты $h_{\alpha \beta}(J, \varphi)(4.1)$ гладкого инвариантного тензора $h$ типа $(0,2)$ ограничены по модулю на любом торе $\mathbb{T}^{k}(2.4)$. Решения (4.5) ограничены при всех $t$ тогда и только тогда, если

$$
\widetilde{b}_{2}(J)=-\widetilde{b}_{3}(J), \quad \widetilde{b}_{4}(J)=0 .
$$

Отсюда следует блочный вид (4.2).

Типичные траектории $C$-интегрируемой невырожденной гамильтоновой системы (1.1) всюду плотны на торах (2.4). Поэтому формулы (4.5) и (4.6) доказывают, что компоненты $h_{\alpha \beta}(4.1)$ зависят только от координат $J_{i}$.

СлеДСТвИЕ 4.1. Любой С-инвариантный тензор $h$ типа $(0,2)$ удовлетворяет уравнению

$$
h(D u, v)+h(u, D v)=0
$$

и аннулирует $k$-мерное распределение $\mathscr{L}_{x}(2.5): h\left(\mathscr{L}_{x}, \mathscr{L}_{x}\right)=0$.

Доказательство следует из формул (2.12) и (4.2). 
II. ТЕОрема 1, ЧАСТь 2. 1) Любъе две симметрии $U$ и $U_{1}$ C-интегрируемой невырожденной гамильтоновой системы (1.1) и любой $C$-инвариантный тензор $h$ типа $(0,2)$ удовлетворяют уравнениям

$$
h\left(U, U_{1}\right)=0, \quad \operatorname{rank}\left(L_{U} h\right) \leqslant k, \quad L_{U_{1}} L_{U} h=0 .
$$

2) Симплектическая структура $\omega_{1}=P_{1}^{-1}$ С-интегрируемой невырожденной гамильтоновой системы (1.1) удовлетворяет уравнениям

$$
\operatorname{rank}\left(L_{U} \omega\right) \leqslant k, \quad L_{U} L_{U} \omega=0
$$

где $U-$ произвольная симметрия системы (1.1).

ДокАзАтЕЛьство. 1) Первое уравнение (4.7) следует из блочной структуры (4.2) и формулы (2.15). Производная Ли $L_{U} h$ имеет вид

$$
\left(L_{U} h\right)_{\alpha \beta}=\dot{h}_{\alpha \beta}+U_{, \alpha}^{\gamma} h_{\gamma \beta}+U_{, \beta}^{\gamma} h_{\alpha \gamma}
$$

Используя формулы (2.16) и (4.2), мы находим, что тензор (4.9) имеет блочную структуру

$$
\left(L_{U} h\right)_{\alpha \beta}=\left(\begin{array}{cc}
\widetilde{a}(J) & 0 \\
0 & 0
\end{array}\right),
$$

где $\widetilde{a}(J)_{i j}=U_{, j}^{\ell+k} b_{i \ell}-U_{, i}^{\ell+k} b_{\ell j}$. Отсюда следует второе уравнение (4.7). Применяя производную Ли $L_{U_{1}}$ к тензору $L_{U} h(4.10)$ типа $(0,2)$, мы вьводим третье уравнение (4.7).

2) Гамильтонова система (1.1) сохраняет симплектическую структуру $\omega$. Поэтому (4.8) следуют из (4.7).

\section{$\S 5$. Любая $C$-инвариантная метрика является неопределенной}

$C$-инвариантный тензор $h_{\alpha \beta}(4.2)$ типа $(0,2)$ очевидно является симметричным, если выполняются уравнения

$$
a^{t}=a, \quad b^{t}=-b .
$$

Обозначим через $g_{\alpha \beta}$ возникающую метрику на многообразии $M^{2 k}$.

ТЕорема 1, чАСТь 3. 1) Любая С-инвариантная метрика $g_{\alpha \beta}=g_{\beta \alpha}$ является неопределенной с сигнатурой $\sigma=\left|n_{+}-n_{-}\right| \leqslant k$. Числа $n_{+} u n_{-}$ее положстельных и отрицательных квадратов удовлетворяют неравенствам

$$
n_{+} \leqslant k, \quad n_{-} \leqslant k
$$

Любая $C$-инвариантная метрика $g_{\alpha \beta}$ nри $n=4 \ell+2$ является вырожденной всюду на многообразии $M^{n}$.

2) Траектории С-интегрируемой гамильтоновой системы (1.1) и траектории любого $C$-инвариантного векторного поля $U$ являются световими геодезическими любой невырожденной $C$-инвариантной метрики $g_{\alpha \beta}$ при $n=4 \ell$. 
ДокАЗАТЕЛЬСтво. 1) Предложение 4.1 доказывает, что $C$-инвариантная метрика $g_{\alpha \beta}$ имеет блочный вид (4.2), где матрица $a(J)$ симметрична и матрица $b(J)$ кососимметрична вследствие (5.1). Поэтому ограничение метрики (4.2) на $k$-мерное распределение $\mathscr{L}_{x} \subset T_{x}\left(M^{2 k}\right)$ равно нулю и неравенства (5.2) справедливы вместе с $\sigma=\left|n_{+}-n_{-}\right| \leqslant k$.

Для любого нечетного $k=2 \ell+1$, кососимметричная $k \times k$ матрица $b(J)$ является вырожденной. Формула (4.2) влечет

$$
\operatorname{det}\|g\|=(\operatorname{det}\|b\|)^{2}=0
$$

Следовательно, при $n=2 k=4 \ell+2$ любая $C$-инвариантная метрика $g$ вырождена.

2) Траектории системы (1.1) и траектории любого $C$-инвариантного векторного поля $U(2.15)$ имеют вид

$$
\begin{array}{ll}
J_{i}=\text { const }, & \varphi_{i}(t)=J_{i} t+\varphi_{i}(0), \\
J_{i}=\text { const }, \quad \varphi_{i}(t)=U^{i+k}(J) t+\varphi_{i}(0)
\end{array}
$$

в тороидальных координатах $x^{i}=J_{i}, x^{i+k}=\varphi_{i}$. Траектории (5.3) очевидно удовлетворяют уравнениям

$$
\frac{d x^{i}(t)}{d t}=0, \quad \frac{d x^{i+k}(t)}{d t}=\mathrm{const}, \quad 1 \leqslant i \leqslant k, \quad \frac{d^{2} x^{\alpha}(t)}{d t^{2}}=0 .
$$

Вследствие блочной структуры (4.2), траектории (5.3) имеют нулевую длину (или являются "световыми” траекториями) по отношению к любой $C$-инвариантной метрике $g$.

Кривая $x^{\alpha}(t)$ является геодезической метрики $g_{\alpha \beta}$, если она удовлетворяет уравнениям

$$
\frac{d^{2} x^{\alpha}}{d t^{2}}+\Gamma_{\beta \gamma}^{\alpha} \frac{d x^{\beta}}{d t} \frac{d x^{\gamma}}{d t}=0 .
$$

Здесь символы Кристоффеля $\Gamma_{\beta \gamma}^{\alpha}$ имеют вид

$$
\Gamma_{\beta \gamma}^{\alpha}=\frac{1}{2} g^{\alpha \tau}\left(\frac{\partial g_{\tau \gamma}}{\partial x^{\beta}}+\frac{\partial g_{\beta \tau}}{\partial x^{\gamma}}-\frac{\partial g_{\beta \gamma}}{\partial x^{\tau}}\right)
$$

Вследствие (5.4), ненулевые слагаемые в (5.5) имеют коэффициенты

$$
\Gamma_{i+k . j+k}^{\alpha}=\frac{1}{2} g^{\alpha \tau}\left(\frac{\partial g_{\tau . j+k}}{\partial x^{i+k}}+\frac{\partial g_{i+k . \tau}}{\partial x^{j+k}}-\frac{\partial g_{i+k . j+k}}{\partial x^{\tau}}\right) .
$$

Используя формулу (4.2), мы находим что все коэффициенты (5.6) равны нулю. Поэтому траектории (5.3)-(5.4) удовлетворяют уравнениям (5.5) и параметр $t$ в (5.3) является аффинным параметром. Следовательно, траектории (5.3) являются световыми геодезическими любой $C$-инвариантной невырожденной метрики. 


\section{§6. Классификация $C$-инвариантных замкнутых дифференциальных 2-форм}

I. $C$-инвариантный тензор $h_{\alpha \beta}(4.2)$ типа $(0,2)$ очевидно является кососимметричным, если справедливы уравнения

$$
a^{t}=-a, \quad b^{t}=b .
$$

Соответствующая дифференциальная 2-форма $\omega_{2}$ имеет вид

$$
\omega_{2}=a_{i \ell}(J) d J_{i} \wedge d J_{\ell}+2 b_{i \ell}(J) d J_{i} \wedge d \varphi_{\ell} .
$$

В статьях [1], [3] мы представили полную классификацию $C$-инвариантных замкнутых 2-форм. В данном параграфе дается другое доказательство этой классификации.

ПРЕДЛОЖЕНИЕ 6.1. Замкнутая Әифференциальная 2-форма $\omega_{2}$ является инвариантной по отношению $\kappa$-интегрируемой невырожденной гамильтоновой системе (1.1) тогда и только тогда, если она имеет вид

$$
\omega_{2}=d\left(\frac{\partial B(J)}{\partial J_{i}}\right) \wedge d \varphi_{i}+d f_{i}(J) \wedge d J_{i}
$$

в тороидальных координатах (2.7). Здесь $B(J)$ и $f_{i}(J)$ являются произвольными гладкими функииями $k$ переменных $J_{1}, \ldots, J_{k}$.

ДокАЗАТЕЛЬСТво. Производная Ли от 2-формы $\omega_{2}$ по отношению к динамической системе (2.6) имеет вид

$$
\dot{\omega}_{2}=d\left(\frac{\partial B(J)}{\partial J_{i}}\right) \wedge d J_{i}=\frac{\partial^{2} B(J)}{\partial J_{i} \partial J_{\ell}} d J_{\ell} \wedge d J_{i}=0 .
$$

Поэтому замкнутая 2-форма (6.3) является $C$-инвариантной.

Вследствие предложения 4.1 и уравнений (6.1) любая $C$-инвариантная 2-форма $\omega_{2}$ имеет вид (6.2). Уравнение $d \omega_{2}=0$ эквивалентно системе $k+1$ уравнений

$$
d\left(a_{i \ell}(J) d J_{i} \wedge d J_{\ell}\right)=0, \quad d\left(b_{i \ell}(J) d J_{i}\right)=0, \quad \ell=1, \ldots, k .
$$

Применяя лемму Пуанкаре, мы получаем

$$
\begin{aligned}
a_{i \ell}(J) d J_{i} \wedge d J_{\ell} & =d\left(f_{i}(J) d J_{i}\right), \\
2 b_{i \ell}(J) d J_{i} & =d B_{\ell}(J),
\end{aligned}
$$

где $f_{i}(J)$ и $B_{\ell}(J)$ - некоторыегладкие функции. Уравнения (6.5) и симметричность матрицы $b(6.1)$ влекут

$$
2 b_{i \ell}=2 b_{\ell i}=\frac{\partial B_{\ell}(J)}{\partial J_{i}}=\frac{\partial B_{i}(J)}{\partial J_{\ell}} .
$$

Отсюда следуют равенства

$$
B_{i}(J)=\frac{\partial B(J)}{\partial J_{i}}, \quad 2 b_{i \ell}=\frac{\partial^{2} B(J)}{\partial J_{i} \partial J_{\ell}}
$$

где $B(J)$ - некоторая гладкая функция.

Формула (6.3) следует из (6.2) после подстановки выражений (6.4) и (6.6).

Предложения 3.1 и 6.1 приводят к следствию: 
Если функиия $B(J)$ нелинейна, то С-инвариантная замкнутая 2-форма $\omega_{2}(6.3)$ не является внешней производной никакой $C$-инвариантной 1-формы $\theta(3.2)$.

II. Применим оператор $i_{V}$ к $C$-инвариантной 2-форме $\omega_{2}$ (6.3). По определению [15] мы имеем $i_{V} \omega_{2}(X)=2 \omega_{2}(V, X)$. Используя формулы (3.3) и $(6.3)$, получаем

$$
i_{V} \omega_{2}=-J_{i} \frac{\partial^{2} B(J)}{\partial J_{i} \partial J_{\ell}} d J_{\ell}=d\left(B(J)-J_{i} \frac{\partial B(J)}{\partial J_{i}}\right) .
$$

Эта формула означает, что 1-форма $i_{V} \omega_{2}$ является дифференциалом первого интеграла

$$
F(J)=B(J)-J_{i} \frac{\partial B(J)}{\partial J_{i}}
$$

гамильтоновой системы (1.1)-(2.6). Первый интеграл $F(J)$ совпадает с преобразованием Лежандра функции $-B(J)$.

\section{§ 7. Пуассоновы структуры и $C$-инвариантные тензоры типа $(2,0)$}

I. Представим тензор $P^{\alpha \beta}(J, \varphi)$ типа $(2,0)$ в блочном виде

$$
P^{\alpha \beta}(J, \varphi)=\left(\begin{array}{ll}
p_{4} & p_{3} \\
p_{2} & p_{1}
\end{array}\right)
$$

в тороидальных координатах $J_{i}, \varphi_{i}$. Здесь $p_{1}, p_{2}, p_{3}, p_{4}(J, \varphi)$ - матрицы размера $k \times k$.

ПрЕДЛОЖЕНИЕ 7.1. Тензор $P^{\alpha \beta}$ типа $(2,0)$ тогда и только тогда является инвариантным по отношению $к$ С-интегрируемой невырожсденной гамильтоновой системе (1.1), если он имеет блочный вид

$$
P^{\alpha \beta}=\left(\begin{array}{cc}
0 & -p(J) \\
p(J) & p_{1}(J)
\end{array}\right)
$$

в координатах $J_{i}, \varphi_{i}$. Здесь $p_{1}(J)$ u $p(J)$ - произвольные $k \times k$ матрицы, зависящие от переменных $J$.

ДоказАТЕЛЬСтво. Уравнение инвариантности $L_{V} P=0$ для тензора $P$ типа $(2,0)$ имеет вид

$$
\left(L_{V} P\right)^{\alpha \beta}=\dot{P}^{\alpha \beta}-V_{, \gamma}^{\alpha} P^{\gamma \beta}-V_{, \gamma}^{\beta} P^{\alpha \gamma}=0 .
$$

После подстановки формул (3.4) и (7.1), уравнение (7.3) принимает эквивалентный вид

$$
\dot{p}_{1}=p_{2}+p_{3}, \quad \dot{p}_{2}=p_{4}, \quad \dot{p}_{3}=p_{4}, \quad \dot{p}_{4}=0,
$$

где $p_{1}, p_{2}, p_{3}, p_{4}-k \times k$ матрицы (7.1). Вследствие (2.8), все решения линейной верхнетреугольной системы (7.4) даются формулами

$$
\begin{gathered}
p_{1}(t)=\widetilde{p}_{4}(J) t^{2}+\left(\widetilde{p}_{2}(J)+\widetilde{p}_{3}(J)\right) t+\widetilde{p}_{1}(J), \\
p_{2}(t)=\widetilde{p}_{4}(J) t+\widetilde{p}_{2}(J), \quad p_{3}(t)=\widetilde{p}_{4}(J) t+\widetilde{p}_{3}(J), \quad p_{4}(t)=\widetilde{p}_{4}(J) .
\end{gathered}
$$

Все компонентыгладкого $C$-инвариантного тензора $P^{\alpha \beta}$ ограничены по модулю на любом торе (2.4). Решения (7.5) ограничены при всех $t$ тогда и только тогда, если

$$
\widetilde{p}_{2}(J)=-\widetilde{p}_{3}(J), \quad \widetilde{p}_{4}(J)=0 .
$$


Следовательно, используя (7.5) и тот факт, что общие траектории невырожденных интегрируемых систем (1.1), (2.6) всюду плотны на торах $\mathbb{T}^{k}$, мы получаем блочную структуру (7.2).

II. Скобкой Схоутена двух кососимметричных тензоров $P$ и $Q$ типа $(2,0)$ называется кососимметричный тензор типа $(3,0)$, компоненты которого имеют вид

$$
\begin{aligned}
2[P, Q]^{\alpha \beta \gamma}= & P_{, \tau}^{\alpha \beta} Q^{\tau \gamma}+P_{, \tau}^{\beta \gamma} Q^{\tau \alpha}+P_{, \tau}^{\gamma \alpha} Q^{\tau \beta} \\
& +Q_{, \tau}^{\alpha \beta} P^{\tau \gamma}+Q_{, \tau}^{\beta \gamma} P^{\tau \alpha}+Q_{, \tau}^{\gamma \alpha} P^{\tau \beta} .
\end{aligned}
$$

Тензор $P^{\alpha \beta}$ типа $(2,0)$ называется пуассоновой структурой, если он кососимметричен, $P^{\alpha \beta}=-P^{\beta \alpha}$, и его скобка Схоутена с самим собой равна нулю

$$
[P, P]^{\alpha \beta \gamma}=P_{, \tau}^{\alpha \beta} P^{\tau \gamma}+P_{, \tau}^{\beta \gamma} P^{\tau \alpha}+P_{, \tau}^{\gamma \alpha} P^{\tau \beta}=0 .
$$

Две пуассоновы структуры $P$ и $Q$ называются совместимыми в смысле Магри [19], если их скобка Схоутена равна нулю $[P, Q]=0$.

ПреДЛОЖЕНИЕ 7.2. Тензор $P^{\alpha \beta}$ mипа $(2,0)$ является $C$-инвариантной пуассоновой структурой тогда и только тогда, если он имеет блочную структуру (7.2) в координатах (2.7) и $k \times k$ матрицы $p(J)$ и $p_{1}(J)$ удовлетворяют уравнениям

$$
\begin{gathered}
p^{t}=p, \quad p_{1}^{t}=-p_{1}, \\
p_{, m}^{i j} p^{m \ell}=p_{, m}^{i \ell} p^{m j}, \\
p_{1, m}^{i j} p^{m \ell}+p_{1, m}^{j \ell} p^{m i}+p_{1, m}^{\ell i} p^{m j}=0 .
\end{gathered}
$$

ДокаЗАТЕЛЬство. Формулы (7.8) следуют из (7.2) и уравнений $P^{\alpha \beta}=-P^{\beta \alpha}$. Формулы (7.9) эквивалентны (7.7) для индексов $(\alpha, \beta, \gamma)=(i, j+k, \ell+k)$. Для остальных значений индексов $(\alpha, \beta, \gamma)=(i, j, \ell)$ или $(\alpha, \beta, \gamma)=(i, j, \ell+k)$, формулы (7.7) справедливы тождественно для любых тензоров (7.2).

III. ТЕОрема 1, часть 4. 1) Любъе две симметрии $U$ и $U_{1}$ C-интегрируемой невырожденной гамильтоновой системъ (1.1) и любой $C$-инвариантный тензор $P$ типа $(2,0)$ удовлетворяют уравнениям

$$
\operatorname{rank}\left(L_{U} P\right) \leqslant k, \quad L_{U_{1}} L_{U} P=0 .
$$

2) Любой $C$-инвариантный тензор $P$ типа $(2,0)$ аннулирует любъе две $C$-инвариантные 1-формы $\theta_{1}, \theta_{2}: P\left(\theta_{1}, \theta_{2}\right)=0$.

3) Любой $C$-инвариантный тензор $P$ mипа $(2,0)$ и любая симметрия $U$ удовлетворяют уравнению

$$
\left[L_{U} P, L_{U} P\right]=0
$$

Уравнение (7.12) означает, что кососимметричный тензор $L_{U} P$ типа $(2,0)$ является (С-инвариантной) пуассоновой структурой. Если тензор $P$ типа $(2,0)$ является пуассоновой структурой, то $P$ и $L_{U} P$ совместимы в смысле Магрu.

4) Для любъх двух $C$-инвариантных кососимметричных тензоров $P$ и $Q$ типа $(2,0)$ и любых двух симметрий $U$ и $U_{1}$ кососимметричные тензорь $L_{U} P$ u $L_{U_{1}} Q$ типа $(2,0)$ являются пуассоновыми структурами и совместимы в смыссле Магри. 
ДокАЗАТЕЛЬСТво. 1) Производная Ли $L_{U} P$ имеет вид

$$
\left(L_{U} P\right)^{\alpha \beta}=\dot{P}^{\alpha \beta}-U_{, \gamma}^{\alpha} P^{\gamma \beta}-U_{, \gamma}^{\beta} P^{\alpha \gamma}
$$

Используя формулы (2.16) и (7.2), мы получаем, что тензор (7.13) имеет блочную структуру

$$
\left(L_{U} P\right)^{\alpha \beta}=\left(\begin{array}{cc}
0 & 0 \\
0 & \widetilde{p}_{1}(J)
\end{array}\right),
$$

где $\widetilde{p}_{1}(J)^{i j}=U_{, \ell}^{i+k} p(J)^{\ell \cdot j+k}-U_{, \ell}^{j+k} p(J)^{i+k \cdot \ell}$. Отсюда следует первое уравнение (7.11). Применяя производную Ли $L_{U_{1}}$ к тензору $L_{U} P(7.14)$ типа $(2,0)$, мы вьводим второе уравнение (7.11).

2 ) Уравнение $P\left(\theta_{1}, \theta_{2}\right)=0$ следует из формул (3.2) и (7.2).

$3)$ Используя формулу (7.14), легко убедиться, что кососимметричный $C$-инвариантный $(2,0)$ тензор $L_{U} P$ удовлетворяет уравнениям (7.8)-(7.10). Вследствие предложения 7.2 , тензор $L_{U} P$ типа $(2,0)$ является пуассоновой структурой. Поэтому (7.12) справедливо. Если тензор $P$ является пуассоновой структурой, то, применяя производную Ли к скобке Схоутена (7.7), мы получаем

$$
L_{U}[P, P]=2\left[L_{U} P, P\right]=0
$$

Следовательно, две пуассоновы структуры $P$ и $L_{U} P$ совместимы в смысле Магри.

4) Кососимметричные тензоры $L_{U} P$ и $L_{U_{1}} Q$ типа $(2,0)$ являются пуассоновыми структурами вследствие (7.12). Формулы (7.6) и (7.14) приводят к уравнению

$$
\left[L_{U} P, L_{U_{1}} Q\right]=0
$$

Поэтому две пуассоновы структуры $L_{U} P$ и $L_{U_{1}} Q$ совместимы по Магри.

СлеДСТВИЕ 7.1. Пусть $Р$ является произвольной (возможсно вырожсденной) пуассоновой структурой на многообразии $M^{2 k}$. Если гамильтонова система

$$
\dot{x}^{\alpha}=P^{\alpha \beta} H_{, \beta}
$$

является $C$-интегрируемой $и$ невырожденной по отношению $\kappa$ некоторой другой пуассоновой структуре $P_{1}$, то для любой ее симметрии $U$ справедливъг уравнения

$$
\left[L_{U} P, L_{U} P\right]=0, \quad\left[L_{U} P, P\right]=0, \quad L_{U} L_{U} P=0, \quad \operatorname{rank}\left(L_{U} P\right) \leqslant k
$$

ДокАЗАТЕЛЬСтво. Гамильтонова система (7.15) сохраняет пуассонову структуру $P$. Поэтому представленные уравнения следуют из доказанной вьше теоремы 1 , часть 4 .

Любой $C$-инвариантный тензор $P$ типа $(2,0)$ удовлетворяет уравнению

$$
P\left(D u^{*}, v^{*}\right)+P\left(u^{*}, D v^{*}\right)=0
$$

для любых двух ковекторов $u^{*}, v^{*} \in T_{x}^{*}\left(M^{n}\right)$. Действительно, уравнение (7.16) следует из формул (2.13), (3.2) и (7.2). 


\section{§8. Характеристический многочлен любого $C$-инвариантного тензора типа $(1,1)$ является полным квадратом}

I. Представим тензор $A_{\beta}^{\alpha}(J, \varphi)$ типа $(1,1)$ в блочном виде

$$
A_{\beta}^{\alpha}(J, \varphi)=\left(\begin{array}{ll}
B_{2} & B_{4} \\
B_{1} & B_{3}
\end{array}\right)
$$

в тороидальных координатах (2.7). Здесь $B_{1}, B_{2}, B_{3}, B_{4}(J, \varphi)$ - матрицы размера $k \times k$.

ПрЕДЛОЖЕНИЕ 8.1. Тензор $A_{\beta}^{\alpha}(J, \varphi)$ типа $(1,1)$ тогда и только тогда является инвариантным по отношению $к$ С-интегрируемой невырожденной гамильтоновой системе (1.1), если он имеет блочный вид

$$
A_{\beta}^{\alpha}=\left(\begin{array}{cc}
B(J) & 0 \\
\sigma(J) & B(J)
\end{array}\right)
$$

в координатах (2.7). Здесь $B(J)$ и $\sigma(J)-$ произвольные $k \times k$ матрицы.

ДоказАтЕльство. Уравнение инвариантности $L_{V} A=0$ для тензора $A_{\beta}^{\alpha}(J, \varphi)$ (8.1) типа $(1,1)$ имеет вид

$$
\left(L_{V} A\right)_{\beta}^{\alpha}=\dot{A}_{\beta}^{\alpha}-V_{, \gamma}^{\alpha} A_{\beta}^{\gamma}+V_{, \beta}^{\gamma} A_{\gamma}^{\alpha}=0
$$

Используя формулы (8.1) и (3.4), мы находим, что уравнение инвариантности (8.3) эквивалентно следующей линейной динамической системе в пространстве матрищ

$$
\dot{B}_{1}=B_{2}-B_{3}, \quad \dot{B}_{2}=-B_{4}, \quad \dot{B}_{3}=B_{4}, \quad \dot{B}_{4}=0 .
$$

Эта система очевидно имеет верхне-треугольную структуру. Вследствие (2.8), решения уравнения (8.4) имеют вид

$$
\begin{gathered}
B_{1}(t)=-\widetilde{B}_{4}(J) t^{2}+\left(\widetilde{B}_{2}(J)-\widetilde{B}_{3}(J)\right) t+\widetilde{B}_{1}(J), \\
B_{2}(t)=-\widetilde{B}_{4}(J) t+\widetilde{B}_{2}(J), \quad B_{3}(t)=\widetilde{B}_{4}(J) t+\widetilde{B}_{3}(J), \quad B_{4}(t)=\widetilde{B}_{4}(J) .
\end{gathered}
$$

Все компоненты гладкого инвариантного $(1,1)$ тензора $A_{\beta}^{\alpha}(J, \varphi)$ ограничены по модулю на любом торе $\mathbb{T}^{k}(2.4)$. Решения (8.5) ограничены при всех $t$ тогда и только тогда, если

$$
\widetilde{B}_{2}(J)=\widetilde{B}_{3}(J), \quad \widetilde{B}_{4}(J)=0 .
$$

Поэтому, используя тот факт, что общие траектории интегрируемой невырожденной гамильтоновой системы $(1,1)$ всюду плотны на торах $\mathbb{T}^{k}$, мы получаем, что любой $C$-инвариантный тензор $A_{\beta}^{\alpha}$ типа $(1,1)$ имеет блочный вид $(8.2)$. 
II. Теорема 1, ЧАСТь 5. 1) Если тензор $A_{\beta}^{\alpha}(x)$ типа $(1,1)$ является $C$-инвариантным по отношению $\kappa$ С-интегрируемой гамильтоновой системе $(1,1)$, то его характеристический многочлен

$$
P(\lambda, x)=\operatorname{det}\|A(x)-\lambda\|
$$

является полным квадратом

$$
P(\lambda, x)=Q^{2}(\lambda, x) .
$$

Все собственнше значения любого $C$-инвариантного тензора типа $(1,1)$ имеют четные кратности.

2) Любъе две симметрии $U$ и $U_{1}$ C-интегрируемой гамильтоновой системьи (1.1) и любой $C$-инвариантный тензор $A(x)$ удовлетворяют уравнениям

$$
\left(L_{U} A\right)^{2}=0, \quad \operatorname{rank}\left(L_{U} A\right) \leqslant k, \quad L_{U_{1}} L_{U} A=0 .
$$

ДокАЗАТЕЛЬство. 1) Характеристический многочлен (8.6) является инвариантом тензора $A_{\beta}^{\alpha}$ типа $(1,1)$ и не зависит от выбора локальных координат. Используя блочную структуру $(8.2) C$-инвариантного тензора $A_{\beta}^{\alpha}$ типа $(1,1)$ в тороидальных координатах (2.7), мы получаем формулу (8.7), где

$$
Q(\lambda, x)=\operatorname{det}\|B(J)-\lambda\| .
$$

Формула (8.7) доказывает, что каждый корень многочлена $P(\lambda, x)$ или каждое собственное значение тензора $A_{\beta}^{\alpha}$ типа $(1,1)$ имеют четную кратность.

2) Производная Ли $L_{U} A$ имеет следующие компоненты

$$
\left(L_{U} A\right)_{\beta}^{\alpha}=\dot{A}_{\beta}^{\alpha}-U_{, \gamma}^{\alpha} A_{\beta}^{\gamma}+U_{, \beta}^{\gamma} A_{\gamma}^{\alpha} .
$$

Используя формулы $(2.16)$ и $(8.2)$, мы получаем, что тензор $(8.9)$ типа $(1,1)$ имеет блочную структуру

$$
L_{U} A=\left(\begin{array}{cc}
0 & 0 \\
C & 0
\end{array}\right)
$$

где $k \times k$ матрица $C(J)$ имеет компоненты $C_{j}^{i}=B_{\ell}^{i} U_{, j}^{\ell+k}-U_{, \ell}^{i+k} B_{j}^{\ell}$. Тензор (8.10) типа $(1,1)$ очевидно удовлетворяет первому и второму уравнениям $(8.8)$.

Применяя производную Ли $L_{U_{1}}$ к тензору $L_{U} A(8.10)$ типа $(1,1)$, мы выводим третье уравнение (8.8).

СлЕДСТВИЕ 8.1. 1) Нильпотентный тензор $D(2.12)$ типа $(1,1)$ является С-инвариантным.

2) Любой $C$-инвариантный тензор $A$ типа $(1,1)$ коммутирует с нильпотентным тензором $D(2.12)$

$$
A D=D A .
$$

Распределение $\mathscr{L}_{x}=T_{x}\left(\mathbb{T}^{k}\right)(2.5)$ инвариантно по отношению $к$ любому $C$-инвариантному тензору А типа $(1,1)$ :

$$
A\left(\mathscr{L}_{x}\right) \subset \mathscr{L}_{x}
$$

ДокаЗАТЕЛЬСтво. 1) Формулы $(2.12)$ означают что тензор $D$ типа $(1,1)$ имеет блочную структуру $(8.2)$, где $B(J)=0$ и $\sigma(J)=1$. Поэтому инвариантность тензора $D$ следует из предложения 8.1.

2) Уравнения (8.11) и (8.12) следуют из блочной структуры (8.2) и формул (2.12). 


\section{§9. Тензор Нейенхейса и $C$-инвариантные тензоры типа $(1,2)$}

I. Тензор Нейенхейса $N_{A \beta \gamma}^{\alpha}$ типа $(1,2)$ определяется формулой [14]

$$
N_{A \beta \gamma}^{\alpha}=A_{\gamma, \tau}^{\alpha} A_{\beta}^{\tau}-A_{\beta, \tau}^{\alpha} A_{\gamma}^{\tau}+\left(A_{\beta, \gamma}^{\tau}-A_{\gamma, \beta}^{\tau}\right) A_{\tau}^{\alpha} \text {. }
$$

Тензор Нейенхейса $N_{A}$ является $C$-инвариантным, если тензор $A$ типа $(1,1) C$-инвариантен. Тензор Нейенхейса $N_{A}$ обладает замечательными алгебраическими свойствами, которые могут быть выведены прямым вычислением, используя определение этого тензора (9.1) и формулу (8.2). Это вычисление показывает, что основные алгебраические свойства тензора Нейенхейса $N_{A}$ являются проявлениями общих алгебраических свойств всех $C$-инвариантных тензоров $N_{\beta \gamma}^{\alpha}$ типа $(1,2)$.

II. Любой тензор $N_{\beta \gamma}^{\alpha}$ типа $(1,2)$ определяет билинейное произведение касательных векторов $u, v \in T_{x}\left(M^{n}\right)$

$$
(N(u, v))^{\alpha}=N_{\beta \gamma}^{\alpha} u^{\beta} v^{\gamma} \in T_{x}\left(M^{n}\right) .
$$

Эта формула определяет некоторую алгебраическую структуру в касательном пространстве $T_{x}\left(M^{n}\right)$.

Мы объединяем компоненты тензора $N_{\beta \gamma}^{\alpha}(J, \varphi)$ в тороидальных координатах $J_{i}, \varphi_{i}(2.7)$ в следуюшие восемь групा

$$
\begin{gathered}
N_{j \ell}^{i+k}=N_{1 j \ell}^{i}, \quad N_{j+k . \ell}^{i+k}=N_{2 j \ell \ell}^{i}, \quad N_{j . \ell+k}^{i+k}=N_{3 j \ell}^{i}, \quad N_{j \ell}^{i}=N_{4 j \ell}^{i}, \\
N_{j+k . \ell}^{i}=N_{5 j \ell}^{i}, \quad N_{j . \ell+k}^{i}=N_{6 j \ell}^{i}, \quad N_{j+k . \ell+k}^{i+k}=N_{7 j \ell}^{i}, \quad N_{j+k . \ell+k}^{i}=N_{8 j \ell}^{i},
\end{gathered}
$$

где индексы $i, j, \ell$ изменяются между 1 и $k$.

ПрЕДЛОЖЕНИЕ 9.1. Тензор $N_{\beta \gamma}^{\alpha}(J, \varphi)$ типа $(1,2)$ является инвариантным по отношению $к$ С-интегрируемой невырожденной гамильтоновой системе (1.1) тогда и только тогда, если все компоненты (9.3) зависят только от переменных $J_{i}$ и справедливы уравнения

$$
N_{4}(J)=N_{2}(J)+N_{3}(J), \quad N_{5}=N_{6}=N_{7}=N_{8}=0 .
$$

Компоненты групп $N_{1}(J), N_{2}(J)$ и $N_{3}(J)$ являются произвольными гладкими функциями от $J_{1}, \ldots, J_{k}$.

ДокАЗАТЕльСтво. Уравнение инвариантности $L_{V} N=0$ для тензора $N_{\beta \gamma}^{\alpha}(J, \varphi)$ типа $(1,2)$ имеет вид

$$
\left(L_{V} N\right)_{\beta \gamma}^{\alpha}=\dot{N}_{\beta \gamma}^{\alpha}-V_{, \tau}^{\alpha} N_{\beta \gamma}^{\tau}+V_{, \beta}^{\tau} N_{\tau \gamma}^{\alpha}+V_{, \gamma}^{\tau} N_{\beta \tau}^{\alpha}=0 .
$$

Подставляя формулы (3.4) и (9.3), мы находим, что уравнения (9.5) эквивалентны следуюшей линейной динамической системе в пространстве тензоров $N_{a}$, $a=1, \ldots, 8$ :

$$
\begin{gathered}
\dot{N}_{1}=N_{4}-N_{2}-N_{3}, \quad \dot{N}_{2}=N_{5}-N_{7}, \quad \dot{N}_{3}=N_{6}-N_{7}, \\
\dot{N}_{4}=-N_{5}-N_{6}, \quad \dot{N}_{5}=-N_{8}, \quad \dot{N}_{6}=-N_{8}, \quad \dot{N}_{7}=N_{8}, \quad \dot{N}_{8}=0 .
\end{gathered}
$$


Используя очевидную верхнетреугольную структуру системы (9.6) и основное свойство первых интегралов (2.8), мы получаем явные формулы для всех решений системы (9.6):

$$
\begin{gathered}
N_{1}(t)=\widetilde{N}_{8}(J) t^{3}+\left(\widetilde{N}_{7}(J)-\widetilde{N}_{5}(J)-\widetilde{N}_{6}(J)\right) t^{2} \\
+\left(\widetilde{N}_{4}(J)-\widetilde{N}_{2}(J)-\widetilde{N}_{3}(J)\right) t+\widetilde{N}_{1}(J), \\
N_{2}(t)=-\widetilde{N}_{8}(J) t^{2}+\left(\widetilde{N}_{5}(J)-\widetilde{N}_{7}(J)\right) t+\widetilde{N}_{2}(J), \\
N_{3}(t)=-\widetilde{N}_{8}(J) t^{2}+\left(\widetilde{N}_{6}(J)-\widetilde{N}_{7}(J)\right) t+\widetilde{N}_{3}(J), \\
N_{4}(t)=\widetilde{N}_{8}(J) t^{2}-\left(\widetilde{N}_{5}(J)+\widetilde{N}_{6}(J)\right) t+\widetilde{N}_{4}(J), \\
N_{5}(t)=-\widetilde{N}_{8}(J) t+\widetilde{N}_{5}(J), \quad N_{6}(t)=-\widetilde{N}_{8}(J) t+\widetilde{N}_{6}(J), \\
N_{7}(t)=-\widetilde{N}_{8}(J) t+\widetilde{N}_{7}(J), \quad N_{8}(t)=\widetilde{N}_{8}(J),
\end{gathered}
$$

где $\widetilde{N}_{a}(J)$ являются произвольными гладкими функциями от $J_{1}, \ldots, J_{k}$.

Компоненты $N_{\beta \gamma}^{\alpha}(J, \varphi)$ любого гладкого $C$-инвариантного тензора типа $(1,2)$ ограничены по модулю на любом торе $\mathbb{T}^{k}(2.4)$. Решения (9.7) ограничены при всех $t$ тогда и только тогда, если вьполнены уравнения (9.4). Поэтому, используя тот факт, что общие траектории интегрируемой невырожденной гамильтоновой системы (1.1) всюду плотны на торах $\mathbb{T}^{k}$, мы получаем, что все компоненты $N_{\beta \gamma}^{\alpha}$ зависят только от переменных $J_{i}$ и удовлетворяют уравнениям (9.4).

III. Для любого касательного вектора $u \in T_{x}\left(M^{n}\right)$, мы определяем линейный оператор

$$
N_{u}: T_{x}\left(M^{n}\right) \rightarrow T_{x}\left(M^{n}\right), \quad N_{u} w=N(u, w) .
$$

В статье [3], мы ввели следующую полиномиально-значную функцию на касательном расслоении $T\left(M^{n}\right)$

$$
P_{N}(u, \lambda)=\operatorname{det}\left(N_{u}-\lambda\right) .
$$

ПРЕДЛОЖЕНИЕ 9.2. Любой $C$-инвариантнъй тензор $N$ типа $(1,2)$ обладает следующими свойствами.

1) Линейнъе $k$-мернъе подпространства $\mathscr{L}_{x}(2.5)$ являются коммутативными идеалами по отношению к алгебрачческим структурам (9.2):

$$
N\left(\mathscr{L}_{x}, \mathscr{L}_{x}\right)=0, \quad N\left(T_{x}\left(M^{n}\right), \mathscr{L}_{x}\right) \subset \mathscr{L}_{x}, \quad N\left(\mathscr{L}_{x}, T_{x}\left(M^{n}\right)\right) \subset \mathscr{L}_{x} .
$$

2) Многочлен $P_{N}(u, \lambda)$ приводим и является произведением двух многочленов степени $k$. Справедливы тождества

$$
P_{N}(u+v, \lambda)=P_{N}(u, \lambda), \quad P_{N}(v, \lambda)=\lambda^{n}
$$

для произвольных касательных векторов $u \in T_{x}\left(M^{n}\right)$ u $v \in \mathscr{L}_{x}, \mathscr{L}_{x}=$ $T_{x}\left(\mathbb{T}^{k}\right) \subset T_{x}\left(M^{n}\right)$.

3) Нильпотентный $C$-инвариантный $(1,1)$ тензор $D(2.12)$ является дифференцированием С-инвариантной алгебрачческой структуры (9.2), что означает справедливость тождества

$$
D N(u, v)=N(D u, v)+N(u, D v)
$$

для любых касательных векторов $u, v \in T_{x}\left(M^{n}\right)$. 
ДокАЗАТЕЛЬСтво. 1) Касательные векторы $u \in T_{x}\left(M^{n}\right)$ и $v \in \mathscr{L}_{x}$ имеют вид

$$
u=u^{\alpha} e_{\alpha}, \quad v=v^{i+k} e_{i+k}
$$

в базисе (2.9). Поэтому формулы (9.10) являются прямым следствием последних четырех уравнений (9.4).

2) Из формулы (9.10) следует, что линейные операторы $N_{e_{\alpha}}(9.8)$ имеют следуюшую $k \times k$ блочную структуру

$$
N_{e_{i}}=\left(\begin{array}{cc}
V_{i} & 0 \\
U_{i} & W_{i}
\end{array}\right), \quad N_{e_{i+k}}=\left(\begin{array}{cc}
0 & 0 \\
Q_{i} & 0
\end{array}\right) .
$$

Используя формулу $u=u^{i} e_{i}+u^{i+k} e_{i+k}$, мы получаем

$$
P_{N}(u, \lambda)=\operatorname{det}\left(u^{i} V_{i}-\lambda\right) \operatorname{det}\left(u^{i} W_{i}-\lambda\right),
$$

где $i=1, \ldots, k$. Эта формула представляет многочлен $P_{N}(u, \lambda)$ в виде произведения двух сомножителей степени $k$. Тождества (9.11) следуют из (9.13) и (9.14).

3) Тождество (9.12) эквивалентно (9.4). Действительно, эта эквивалентность легко проверяется в базисе (2.9).

Теорема 1, часть 6. 1) Любъе две симметрии $U$ и $U_{1}$ C-интегрируемой невырожденной гамильтоновой системы (1.1) и любые С-инвариантные 1-форма $\theta$ и тензор $N_{\beta \gamma}^{\alpha}$ типа $(1,2)$ удовлетворяют уравнениям

$$
N_{\beta \gamma}^{\alpha} U^{\beta} U_{1}^{\gamma}=0, \quad N_{\beta \gamma}^{\alpha} U^{\beta} \theta_{\alpha}=0, \quad L_{U_{1}} L_{U} N=0 .
$$

2) С-инвариантный тензор $\left(A_{U}\right)_{\beta}^{\alpha}=N_{\beta \tau}^{\alpha} U^{\tau}$ и тензор $\left(N_{\theta}\right)_{\beta \gamma}=N_{\beta \gamma}^{\tau} \theta_{\tau}$ удовлетворяют уравнениям

$$
\begin{gathered}
\operatorname{rank}\left\|A_{U}\right\| \leqslant k, \quad A_{U}^{2}=0, \quad L_{U_{1}} A_{U}=0, \\
\operatorname{rank}\left\|N_{\theta}\right\| \leqslant k, \quad L_{U} N_{\theta}=0 .
\end{gathered}
$$

3) Тензор $\widetilde{N}=L_{U} N$ типа $(1,2)$ определяет нильпотентную алгебраическую структуру в касательном пространстве $T\left(M^{n}\right)$ : уравнения

$$
\widetilde{N}(\widetilde{N}(u, v), w)=0, \quad \widetilde{N}(u, \widetilde{N}(v, w))=0
$$

справедливы для любых касательных векторов $u, v, w \in T_{x}\left(M^{n}\right)$.

4) Многочлен $P_{N}(u, \lambda)(9.9)$ удовлетворяет уравнениям

$$
P_{N}(u+U, \lambda)=P_{N}(u, \lambda), \quad P_{N}(U, \lambda)=\lambda^{n}
$$

для любого касательного вектора $и \in T_{x}\left(M^{n}\right)$ и любой симметрии U. Многочлен $P_{N}(u, \lambda)$ приводим и является произведением двух многочленов степени $k$.

5) Для любых двух $C$-инвариантных тензоров $M$ и $N$ типа $(1,2)$ и любой симметрии $U$, тензор $h_{\alpha \beta}=M_{\alpha \nu}^{\tau} N_{\beta \tau}^{\nu}$ типа $(0,2)$ удовлетворяет уравнениям

$$
\operatorname{rank}\|h\| \leqslant k, \quad h_{\alpha \beta} U^{\beta}=0, \quad L_{U} h=0 .
$$


ДоказАТЕЛЬСтво. 1) Первое уравнение (9.15) следует из первого уравнения (9.10), так как любая симметрия $U(2.15)$ принадлежит распределению $\mathscr{L}_{x}(2.5)$. Второе уравнение (9.15) следует из формул (2.15), (3.2) и (9.4).

Используя предложение 9.1 и формулы (2.16), (2.18) и (9.5), мы получаем, что все ненулевые компоненты тензора $L_{U} N$ типа $(1,2)$ имеют вид

$$
\left(L_{U} N\right)_{j \ell}^{i+k}=U_{, j}^{m+k} N_{m+k . \ell}^{i+k}+U_{, \ell}^{m+k} N_{j . m+k}^{i+k}-U_{, m}^{i+k} N_{j \ell}^{m} .
$$

Поэтому только первая группа (9.3) компонент тензора $\widetilde{N}=L_{U} N$ является ненулевой. Применяя формулу (9.19) к тензору $L_{U_{1}} \widetilde{N}=L_{U_{1}} L_{U} N$ типа $(1,2)$, мы получаем третье уравнение (9.15).

2 ) Вследствие формул (2.15) и (3.2) для симметрий $U$ и для $C$-инвариантных 1-форм $\theta$, и формул $(9.3),(9.4)$, все ненулевые компоненты тензора $A_{U}$ типа $(1,1)$ и тензора $N_{\theta}$ типа $(0,2)$ имеют вид

$$
A_{U . j}^{i+k}=N_{j . \ell+k}^{i+k} U^{\ell+k}, \quad N_{\theta . i j}=N_{i j}^{\ell} \theta_{\ell} .
$$

Отсюда следуют алгебраические уравнения (9.16). Применяя формулы (4.9) и (8.9) к тензорам (9.20), мы выводим уравнения $L_{U_{1}} A_{U}=0, L_{U} N_{\theta}=0$.

3) Мы показали, что все компоненты тензора $\widetilde{N}=L_{U} N$ типа $(1,2)$, за исключением $\left(L_{U} N\right)_{j \ell}^{i+k}$, равны нулю. Этот факт означает, что тензор $\widetilde{N}$ типа $(1,2)$ удовлетворяет уравнениям

$$
\widetilde{N}\left(T_{x}\left(M^{n}\right), T_{x}\left(M^{n}\right)\right) \subset \mathscr{L}_{x}, \quad \widetilde{N}\left(\mathscr{L}_{x}, T_{x}\left(M^{n}\right)\right)=\widetilde{N}\left(T_{x}\left(M^{n}\right), \mathscr{L}_{x}\right)=0 .
$$

Отсюда следуют уравнения (9.17).

4) Утверждение 4) следует из утверждения 2) предложения 9.2 , поскольку векторное поле любой симметрии $U$ принадлежит распределению $\mathscr{L}_{x}=T_{x}\left(\mathbb{T}^{k}\right)$.

$5)$ Используя формулы (9.3) и (9.4) для любых $C$-инвариантных тензоров $M$ и $N$ типа $(1,2)$, легко проверить, что все ненулевые компоненты тензора $h$ типа $(0,2)$ имеют вид

$$
h_{i j}=M_{i . m}^{\ell} N_{j \cdot \ell}^{m}+M_{i . m+k}^{\ell+k} N_{j \cdot \ell+k}^{m+k} .
$$

Отсюда следует первое неравенство (9.18). Второе уравнение (9.18) следует из выражений (2.15) для симметрии $U$ и (9.21). Применяя формулу Лейбница, мы получаем

$$
\left(L_{U} h\right)_{\alpha \beta}=\left(L_{U} M\right)_{\alpha \nu}^{\tau} N_{\beta \tau}^{\nu}+M_{\alpha \nu}^{\tau}\left(L_{U} N\right)_{\beta \tau}^{\nu}
$$

Используя формулы (9.19) для ненулевых компонент тензоров $L_{U} M$ и $L_{U} N$, и формулы (9.3), (9.4), мы находим, что обе суммы в (9.22) равны нулю. Поэтому $L_{U} h=0$ для любой симметрии $U$.

§10. Замкнутые дифференциальные 3 -формы и $C$-инвариантные $(0,3)$ тензоры

I. Мы объединяем компоненты $T_{\alpha \beta \gamma}(J, \varphi)$ тензора $T$ типа $(0,3)$ в следующие восемь групп

$$
\begin{gathered}
T_{i j \ell}=T_{1 i j \ell}, \quad T_{i+k . j . \ell}=T_{2 i j \ell}, \quad T_{i . j+k . \ell}=T_{3 i j \ell}, \\
T_{i . j . \ell+k}=T_{4 i j \ell}, \quad T_{i+k . j . \ell+k}=T_{5 i j \ell}, \quad T_{i+k . j+k . \ell}=T_{6 i j \ell}, \\
T_{i . j+k . \ell+k}=T_{7 i j \ell}, \quad T_{i+k . j+k . \ell+k}=T_{8 i j \ell},
\end{gathered}
$$


где индексы $i, j, \ell$ изменяются между 1 и $k$.

ПрЕДЛОЖЕНИЕ 10.1. Тензор $T_{\alpha \beta \gamma}(J, \varphi)$ muпа $(0,3)$ тогда и только тогда является инвариантным по отношению $\kappa$-интегрируемой невырохденной гамильтоновой системе (1.1), если все компоненты (10.1) зависят только от переменных $J_{i}$ и справедливы уравнения

$$
T_{2}(J)+T_{3}(J)+T_{4}(J)=0, \quad T_{5}=T_{6}=T_{7}=T_{8}=0 .
$$

Компоненты $T_{1}(J), T_{2}(J)$ и $T_{3}(J)$ являются произвольными гладкими функииями переменных $J_{1}, \ldots, J_{k}$.

ДокАЗАТЕЛЬство. Уравнение инвариантности $L_{V} T=0$ для тензора $T_{\alpha \beta \gamma}(J, \varphi)$ типа $(0,3)$ имеет вид

$$
\left(L_{V} T\right)_{\alpha \beta \gamma}=\dot{T}_{\alpha \beta \gamma}+V_{, \alpha}^{\tau} T_{\tau \beta \gamma}+V_{, \beta}^{\tau} T_{\alpha \tau \gamma}+V_{, \gamma}^{\tau} T_{\alpha \beta \tau}=0
$$

Используя формулы (3.4) и (10.1), мы получаем, что уравнения (10.3) эквивалентны следуюшей линейной динамической системе в пространстве тензоров $T_{a}$, $a=1, \ldots, 8$ :

$$
\begin{gathered}
\dot{T}_{1}=-T_{2}-T_{3}-T_{4}, \quad \dot{T}_{2}=-T_{5}-T_{6}, \quad \dot{T}_{3}=-T_{6}-T_{7}, \\
\dot{T}_{4}=-T_{5}-T_{7}, \quad \dot{T}_{5}=-T_{8}, \quad \dot{T}_{6}=-T_{8}, \quad \dot{T}_{7}=-T_{8}, \quad \dot{T}_{8}=0 .
\end{gathered}
$$

Вследствие основного свойства первых интегралов (2.8) и верхне-треугольной структуры системы (10.4), все решения (10.4) определяются формулами

$$
\begin{gathered}
T_{1}(t)=-\widetilde{T}_{8}(J) t^{3}+\left(\widetilde{T}_{5}(J)+\widetilde{T}_{6}(J)+\widetilde{T}_{7}(J)\right) t^{2} \\
-\left(\widetilde{T}_{2}(J)+\widetilde{T}_{3}(J)+\widetilde{T}_{4}(J)\right) t+\widetilde{T}_{1}(J), \\
T_{2}(t)=\widetilde{T}_{8}(J) t^{2}-\left(\widetilde{T}_{5}(J)+\widetilde{T}_{6}(J)\right) t+\widetilde{T}_{2}(J), \\
T_{3}(t)=\widetilde{T}_{8}(J) t^{2}-\left(\widetilde{T}_{6}(J)+\widetilde{T}_{7}(J)\right) t+\widetilde{T}_{3}(J), \\
T_{4}(t)=\widetilde{T}_{8}(J) t^{2}-\left(\widetilde{T}_{5}(J)+\widetilde{T}_{7}(J)\right) t+\widetilde{T}_{4}(J), \\
T_{5}(t)=-\widetilde{T}_{8}(J) t+\widetilde{T}_{5}(J), \quad T_{6}(t)=-\widetilde{T}_{8}(J) t+\widetilde{T}_{6}(J), \\
T_{7}(t)=-\widetilde{T}_{8}(J) t+\widetilde{T}_{7}(J), \quad T_{8}(t)=\widetilde{T}_{8}(J),
\end{gathered}
$$

где $\widetilde{T}_{a}(J)$ являются произвольными гладкими функциями переменных $J_{1}, \ldots, J_{k}$.

Все компоненты $T_{\alpha \beta \gamma}(J, \varphi)$ гладкого $C$-инвариантного тензора $T$ типа $(0,3)$ ограничены по модулю на любом торе $\mathbb{T}^{k}(2.4)$. Решения (10.5) ограничены при всех $t$ тогда и только тогда, если справедливы уравнения (10.2). Поэтому, используя тот факт, что общие траектории интегрируемой невырожденной системы (1.1) всюду плотны на торах $\mathbb{T}^{k}$, мы получаем, что все компоненты $T_{\alpha \beta \gamma}$ зависят только от переменных $J_{i}$ и удовлетворяют уравнениям (10.2). 
Уравнения (10.2) означают, что $C$-инвариантные тензоры $T_{\alpha \beta \gamma}$ типа $(0,3)$ соответствуют особым точкам системы (10.4). Прямая проверка показывает, что уравнения (10.2) эквивалентны тождеству

$$
T(D u, v, w)+T(u, D v, w)+T(u, v, D w)=0,
$$

где $u, v, w \in T_{x}\left(M^{n}\right)$ - произвольные касательные векторы и $D$ - нильпотентньй тензор $(2.12)$ типа $(1,1)$.

II. СлЕДСТвИЕ 10.1. Любая С-инвариантная дифференциальная 3-форма $\omega_{3}$ имеет вид

$$
\omega_{3}=b_{i \ell m}(J) d J_{i} \wedge d J_{\ell} \wedge d \varphi_{m}+c_{i \ell m}(J) d J_{i} \wedge d J_{\ell} \wedge d J_{m},
$$

где коэффициенты $b_{i \ell m}(J)$ удовлетворяют уравнениям

$$
b_{i \ell m}(J)+b_{\ell m i}(J)+b_{m i \ell}(J)=0, \quad b_{i \ell m}(J)=-b_{\ell i m}(J)
$$

и коэффициенты $c_{i \ell m}(J)$ являются знакопеременными.

ДокАЗАТЕЛЬСТво. Формулы (10.6) и (10.7) следуют из (10.2) для любого $C$-инвариантного тензора типа $(0,3)$ и из определения дифференциальных 3 -форм.

ПреДЛОЖЕНИЕ 10.2. 1) Замкнутая дифференциальная 3-форма $\omega_{3}$ тогда и только тогда инвариантна по отношению $к$ С-интегрируемой невырожденной гамильтоновой системе (1.1), если она имеет вид

$$
\omega_{3}=d\left(\frac{\partial B_{i}(J)}{\partial J_{m}}+b_{i m}(J)\right) \wedge d J_{i} \wedge d \varphi_{m}+d\left(a_{i \ell}(J) d J_{i} \wedge d J_{\ell}\right)
$$

в тороидальных координатах (2.7). Здесь коэффициентьи $a_{i \ell}(J)$ и $b_{i m}(J)$ удовлетворяют уравнениям

$$
a_{i \ell}(J)=-a_{\ell i}(J), \quad b_{i m}(J)=b_{m i}(J)
$$

и $B_{i}(J)$ - произвольнье гладкие функиии переменньх $J_{1}, \ldots, J_{k}$.

2) Справедливо уравнение $\omega_{3}=d \widetilde{\omega}_{2}$, әде

$$
\widetilde{\omega}_{2}=\left(\frac{\partial B_{i}(J)}{\partial J_{m}}+\frac{\partial B_{m}(J)}{\partial J_{i}}+b_{i m}(J)\right) d J_{i} \wedge d \varphi_{m}+a_{i \ell}(J) d J_{i} \wedge d J_{\ell}
$$

является С-инвариантной 2-формой.

ДокАЗАТЕльСтво. 1) Производная Ли от замкнутой 3-формы (10.8) по отношению к динамической системе (2.6) имеет вид

$$
\dot{\omega}_{3}=\frac{\partial^{2} B_{i}(J)}{\partial J_{m} \partial J_{\ell}} d J_{\ell} \wedge d J_{i} \wedge d J_{m}+d b_{i m}(J) \wedge d J_{i} \wedge d J_{m}=0 .
$$

Это выражение равно нулю ввиду кососимметричности внешнего произведения и симметричности смешанных вторых производных от функции $B_{i}(J)$ и симметричности коэффициентов $b_{i m}(J)(10.9)$. Поэтому замкнутая 3 -форма $(10.8)$ является $C$-инвариантной. 
Следствие 10.1 показывает, что любая $C$-инвариантная дифференциальная 3 -форма $\omega_{3}$ имеет вид (10.6). Уравнение $d \omega_{3}=0$ эквивалентно $k+1$ уравнениям

$$
\begin{gathered}
d\left(b_{i \ell m}(J) d J_{i} \wedge d J_{\ell}\right)=0, \quad m=1, \ldots, k \\
d\left(c_{i \ell m}(J) d J_{i} \wedge d J_{\ell} \wedge d J_{m}\right)=0 .
\end{gathered}
$$

Применяя лемму Пуанкаре, мы получаем

$$
\begin{gathered}
b_{i \ell m}(J) d J_{i} \wedge d J_{\ell}=d\left(c_{i m}(J) d J_{i}\right), \quad m=1, \ldots, k, \\
c_{i \ell m}(J) d J_{i} \wedge d J_{\ell} \wedge d J_{m}=d\left(a_{i \ell}(J) d J_{i} \wedge d J_{\ell}\right),
\end{gathered}
$$

где $c_{i m}(J)$ и $a_{i \ell}(J)=-a_{\ell i}(J)$ являются гладкими функциями. После подстановки формул (10.11) в (10.6), уравнение инвариантности $\dot{\omega}_{3}=0$ принимает вид

$$
\dot{\omega}_{3}=d\left(c_{i m}(J) d J_{i} \wedge d J_{m}\right)=0 .
$$

Используя лемму Пуанкаре, мы получаем

$$
c_{i m}(J) d J_{i} \wedge d J_{m}=-d\left(B_{i}(J) d J_{i}\right),
$$

где $B_{i}(J)$ - некоторые гладкие функции. Обшее решение уравнения (10.13) имеет вид

$$
c_{i m}(J)=\frac{\partial B_{i}(J)}{\partial J_{m}}+b_{i m}(J), \quad b_{i m}(J)=b_{m i}(J),
$$

где $b_{i m}(J)$ - произвольные гладкие функции от $J_{1}, \ldots, J_{k}$. Теперь формула (10.8) следует из (10.6), (10.11), (10.12) и (10.14) прямой подстановкой.

2) Уравнение $\omega_{3}=d \widetilde{\omega}_{2}$ легко выводится из (10.10). $C$-инвариантность 2-формы $\widetilde{\omega}_{2}$ следует из формулы для производной Ли $L_{V} \widetilde{\omega}_{2}$ :

$$
\begin{aligned}
L_{V} \widetilde{\omega}_{2} & =\dot{\tilde{\omega}}_{2}=\left(\frac{\partial B_{i}(J)}{\partial J_{m}}+\frac{\partial B_{m}(J)}{\partial J_{i}}+b_{i m}(J)\right) d J_{i} \wedge d J_{m} \\
& =d J_{i} \wedge d B_{i}(J)+d B_{m}(J) \wedge d J_{m}+b_{i m}(J) d J_{i} \wedge d J_{m} .
\end{aligned}
$$

Первая и вторая суммы в правой части (10.15) сокращаются. Третья сумма в (10.15) равна нулю ввиду симметричности коэффициентов $b_{i m}(J)$ и кососимметричности внешнего произведения $d J_{i} \wedge d J_{m}$. Отсюда мы получаем $L_{V} \widetilde{\omega}_{2}=0$. Поэтому 2-форма $\widetilde{\omega}_{2}$ является $C$-инвариантной.

Уравнение инвариантности

$$
L_{V} \widetilde{\omega}_{2}=\left(i_{V} \circ d+d \circ i_{V}\right) \widetilde{\omega}_{2}=0
$$

и уравнение $\omega_{3}=d \widetilde{\omega}_{2}$ влекут

$$
i_{V} \omega_{3}=-d\left(i_{V} \widetilde{\omega}_{2}\right)
$$

Поэтому $C$-инвариантная 2-форма $i_{V} \omega_{3}$ является внешней производной $C$-инвариантной 1-формы $-i_{V} \widetilde{\omega}_{2}$. 
III. Теорема 1, часть 7. 1) Любъе две симметрии $U$ и $U_{1} C$-интегрируемой невырожденной гамильтоновой системы (1.1) и любой $C$-инвариантный тензор $T_{\alpha \beta \gamma}$ типа $(0,3)$ удовлетворяют уравнениям

$$
T_{\alpha \beta \gamma} U^{\beta} U_{1}^{\gamma}=0, \quad L_{U_{1}} L_{U} T=0 .
$$

2) С-инвариантный тензор $\left(T_{U}\right)_{\alpha \beta}=T_{\alpha \beta \gamma} U^{\gamma}$ типа $(0,2)$ удовлетворяет уравнениям

$$
\operatorname{rank}\left\|T_{U}\right\| \leqslant k, \quad L_{U_{1}} T_{U}=0 .
$$

ДокАЗАТЕльство. 1) Первое уравнение (10.16) следует из предложения 10.1 и формулы (2.15) для симметрий $U$ в тороидальных координатах (2.7).

Используя предложение 10.1 и формулы $(2.16),(2.18)$ и (10.3), мы получаем, что все ненулевые компоненты тензора $L_{U} T$ типа $(0,3)$ имеют вид

$$
\left(L_{U} T\right)_{i j \ell}=U_{, i}^{m+k} T_{m+k . j . \ell}+U_{, j}^{m+k} T_{i . m+k . \ell}+U_{, \ell}^{m+k} T_{i . j . m+k} .
$$

Поэтому только первая группа компонент (10.1) тензора $\widetilde{T}=L_{U} T$ типа $(0,3)$ отлична от нуля. Применяя формулы $(10.18)$ к тензору $L_{U_{1}} \widetilde{T}=L_{U_{1}} L_{U} T$, мы вьводим второе уравнение (10.16).

2) Вследствие выражения (2.15) для симметрий $U$ и формул (10.1), (10.2), все компоненты тензора $T_{U}$ типа $(0,2)$ имеют вид $T_{U . i j}=T_{i . j . \ell+k} U^{\ell+k}$. Отсюда следует первое уравнение (10.17). Применяя формулы (4.9) к тензору $T_{U}$, мы получаем второе уравнение (10.17).

\section{$\S 11$. Скобки Схоутена и $C$-инвариантные тензоры типа $(3,0)$}

I. Любой тензор $R^{\alpha \beta \gamma}$ типа $(3,0)$ определяет трилинейную функцию на кокасательном пространстве $T_{x}^{*}\left(M^{n}\right)$ :

$$
R(\zeta, \eta, \theta)=R^{\alpha \beta \gamma} \zeta_{\alpha} \eta_{\beta} \theta_{\gamma}
$$

Кососимметричные тензоры $R^{\alpha \beta \gamma}(J, \varphi)$ типа $(3,0)$ имеют важные применения как скобки Схоутена двух общих пуассоновых структур.

Мы объединяем тензорные компоненты $R^{\alpha \beta \gamma}(J, \varphi)$ в тороидальных координатах $J_{i}, \varphi_{i}(2.7)$ в следующие восемь групा

$$
\begin{aligned}
R^{i+k . j+k . \ell+k} & =R_{1}^{i j k}, \quad R^{i . j+k . \ell+k}=R_{2}^{i j k} \\
R^{i+k . j+k . \ell} & =R_{3}^{i j \ell}, \quad R^{i+k . j . \ell+k}=R_{4}^{i j \ell}, \\
R^{i . j . \ell+k}=R_{5}^{i j \ell}, & R^{i . j+k . \ell}=R_{6}^{i j \ell}, \quad R^{i+k . j . \ell}=R_{7}^{i j \ell}, \quad R^{i j \ell}=R_{8}^{i j \ell},
\end{aligned}
$$

где индексы $i, j$ и $\ell$ изменяются между 1 и $k$.

ПрЕДЛОЖЕНИЕ 11.1. Тензор $R^{\alpha \beta \gamma}(J, \varphi)$ типа $(3,0)$ тогда и только тогда инвариантен по отношению $\kappa$ C-интегрируемой невырожденной гамильтоновой системе (1.1), если все компоненть $R^{\alpha \beta \gamma}$ зависят только от переменных $J_{i}$ и справедливы уравнения

$$
R_{4}(J)=-R_{2}(J)-R_{3}(J), \quad R_{5}=R_{6}=R_{7}=R_{8}=0 .
$$

Компоненты $R_{1}(J), R_{2}(J)$ и $R_{3}(J)$ являются произвольньци гладкими функииями переменных $J_{1}, \ldots, J_{k}$. 
ДокАЗАТЕЛЬСтво. Уравнение инвариантности $L_{V} R=0$ имеет вид

$$
\left(L_{V} R\right)^{\alpha \beta \gamma}=\dot{R}^{\alpha \beta \gamma}-V_{, \tau}^{\alpha} R^{\tau \beta \gamma}-V_{, \tau}^{\beta} R^{\alpha \tau \gamma}-V_{, \tau}^{\gamma} R^{\alpha \beta \tau}=0 .
$$

Подставляя формулы (3.4) и (11.1), мы получаем, что уравнения (11.3) эквивалентны следующей линейной динамической системе в пространстве тензоров $R_{a}$, $a=1, \ldots, 8$ :

$$
\begin{aligned}
& \dot{R}_{1}=R_{2}+R_{3}+R_{4}, \quad \dot{R}_{2}=R_{5}+R_{6}, \quad \dot{R}_{3}=R_{6}+R_{7} \\
& \dot{R}_{4}=R_{5}+R_{7}, \quad \dot{R}_{5}=R_{8}, \quad \dot{R}_{6}=R_{8}, \quad \dot{R}_{7}=R_{8}, \quad \dot{R}_{8}=0 .
\end{aligned}
$$

Используя очевидную верхнетреугольную структуру этой системы и основное свойство первых интегралов (2.8), мы представляем все решения уравнений (11.4) в явном виде:

$$
\begin{gathered}
R_{1}(t)=\widetilde{R}_{8}(J) t^{3}+\left(\widetilde{R}_{5}(J)+\widetilde{R}_{6}(J)+\widetilde{R}_{7}(J)\right) t^{2} \\
+\left(\widetilde{R}_{2}(J)+\widetilde{R}_{3}(J)+\widetilde{R}_{4}(J)\right) t+\widetilde{R}_{1}(t), \\
R_{2}(t)=\widetilde{R}_{8}(J) t^{2}+\left(\widetilde{R}_{5}(J)+\widetilde{R}_{6}(J)\right) t+\widetilde{R}_{2}(J), \\
R_{3}(t)=\widetilde{R}_{8}(J) t^{2}+\left(\widetilde{R}_{6}(J)+\widetilde{R}_{7}(J)\right) t+\widetilde{R}_{3}(J), \\
R_{4}(t)=\widetilde{R}_{8}(J) t^{2}+\left(\widetilde{R}_{5}(J)+\widetilde{R}_{7}(J)\right) t+\widetilde{R}_{4}(J), \\
R_{5}(t)=\widetilde{R}_{8}(J) t+\widetilde{R}_{5}(J), \quad R_{6}(t)=\widetilde{R}_{8}(J) t+\widetilde{R}_{6}(J), \\
R_{7}(t)=\widetilde{R}_{8}(J) t+\widetilde{R}_{7}(J), \quad R_{8}(t)=R_{8}(J),
\end{gathered}
$$

где $\widetilde{R}_{a}(J)$ - произвольные гладкие функции переменных $J_{1}, \ldots, J_{k}$.

$C$-инвариантный тензор $R^{\alpha \beta \gamma}(J, \varphi)$ типа $(3,0)$ является гладким в любой тороидальной области $\mathscr{O}=B_{a} \times \mathbb{T}^{k}$. Поэтому все компоненты $R^{\alpha \beta \gamma}(J, \varphi)$ ограничены по модулю на любом торе $\mathbb{T}^{k}$. Точные решения (11.5) ограничены при всех $t$ тогда и только тогда, если справедливы уравнения (11.2). Поэтому, используя тот факт, что обшие траектории интегрируемой невырожденной гамильтоновой системы (1.1) всюду плотны на торах $\mathbb{T}^{k}(2.4)$, мы получаем, что все компоненты $R^{\alpha \beta \gamma}$ удовлетворяют (11.2).

Уравнения (11.2) определяют особые точки динамической системы (11.4). Поэтому тензор $R^{\alpha \beta \gamma}(11.1)$ типа $(3,0)$ является $C$-инвариантным тогда и только тогда, если функции $R_{a}(J)$ зависят только от переменных $J_{i}$ и область их значений принадлежит особому множеству (11.2) динамической системы (11.4). Прямое вычисление доказьвает, что уравнения (11.2) эквивалентны уравнению

$$
R(D \zeta, \eta, \theta)+R(\zeta, D \eta, \theta)+R(\zeta, \eta, D \theta)=0
$$

где $D-C$-инвариантный нильпотентньй тензор $(2.12),(2.13)$ типа $(1,1)$ и $\zeta, \eta, \theta-$ произвольные ковекторы.

II. Теорема 1, часть 8. 1) Любъе две симметрии $U$ и $U_{1} C$-интегрируемой невыродсденной гамильтоновой системы (1.1), любъе две С-инвариантные 1-форми $\theta$ и $\eta$ и любой $C$-инвариантный тензор $R^{\alpha \beta \gamma}$ типа $(3,0)$ удовлетворяют уравнениям

$$
R^{\alpha \beta \gamma} \theta_{\beta} \eta_{\gamma}=0, \quad L_{U_{1}} L_{U} R=0 .
$$


2) С-инвариантный тензор $R_{\theta}^{\alpha \beta}=R^{\alpha \beta \gamma} \theta_{\gamma}$ типа $(2,0)$ удовлетворяет уравнениям

$$
\operatorname{rank}\left\|R_{\theta}\right\| \leqslant k, \quad L_{U} R_{\theta}=0 .
$$

3) Для любых двух C-инвариантных тензоров $R$ и $T$ типа $(0,3)$ и любой симметрии $U$, тензор $A_{\beta}^{\alpha}=R^{\alpha \tau \nu} T_{\beta \tau \nu}$ типа $(1,1)$ удовлетворяет уравнения.M

$$
\operatorname{rank}\|A\| \leqslant k, \quad A^{2}=0, \quad L_{U} A=0
$$

ДокАЗАТЕльство. 1) Первое уравнение (11.6) следует из предложения 11.1 и формул (3.2) для $C$-инвариантных 1-форм.

Вследствие предложения 10.1 и формул $(2.16),(2,18)$ и (11.3), все ненулевые компоненты тензора $L_{U} R$ типа $(3,0)$ имеют вид

$$
\left(L_{U} R\right)^{i+k \cdot j+k \cdot \ell+k}=-U_{, m}^{i+k} R^{m \cdot j+k \cdot \ell+k}-U_{, m}^{j+k} R^{i+k \cdot m \cdot \ell+k}-U_{, m}^{\ell+k} R^{i+k \cdot j+k \cdot m} .
$$

Поэтому только первая группа компонент (11.1) тензора $\widetilde{R}=L_{U} R$ является ненулевой. Применяя формулу (11.9) к тензору $L_{U_{1}} \widetilde{R}=L_{U_{1}} L_{U} R$ типа $(3,0)$, мы получаем второе уравнение (11.6).

$2)$ Вследствие формулы (3.2) для $C$-инвариантных 1-форм и формул (11.1), (11.2), мы получаем, что все ненулевые компоненты тензора $R_{\theta}$ типа $(2,0)$ имеют вид $R_{\theta}^{i+k \cdot j+k}=R^{i+k \cdot j+k \cdot \ell_{\ell}}$. Отсюда следует первое уравнение (11.7). Второе уравнение (11.7) следует из (7.13).

3) Используя формулы (10.2) и (11.2), мы получаем, что все ненулевые компоненты тензора $A$ типа $(1,1)$ имеют вид

$$
A_{j}^{i+k}=R^{i+k . \ell \cdot m+k} T_{j \cdot \ell \cdot m+k}+R^{i+k \cdot \ell+k \cdot m} T_{j \cdot \ell+k \cdot m} .
$$

Поэтому тензор $A$ имеет блочную структуру (8.10). Отсюда следуют первые два уравнения (11.8). Применяя формулу Лейбница, мы находим

$$
\left(L_{U} A\right)_{\beta}^{\alpha}=\left(L_{U} R\right)^{\alpha \tau \nu} T_{\beta \tau \nu}+R^{\alpha \tau \nu}\left(L_{U} T\right)_{\beta \tau \nu} .
$$

Используя формулы (11.9) и (10.18) для ненулевых компонент тензоров $L_{U} R$ и $L_{U} T$, мы получаем, что обе суммы в (11.10) равны нулю. Следовательно, $L_{U} A=0$ для любой симметрии $U$.

\section{$\S 12$. $C$-инвариантные $(2,1)$ тензоры}

I. Любой тензор $S_{\gamma}^{\alpha \beta}$ типа $(2,1)$ определяет алгебраическую структуру в кокасательном пространстве $T_{x}^{*}\left(M^{n}\right)$ :

$$
(S(\zeta, \eta))_{\gamma}=S_{\gamma}^{\alpha \beta} \zeta_{\alpha} \eta_{\beta} \in T_{x}^{*}\left(M^{n}\right) .
$$

Мы объединяем компоненты тензора $S_{\gamma}^{\alpha \beta}(J, \varphi)$ в тороидальных координатах $J_{i}, \varphi_{i}(2.7)$ в следующие восемь групп

$$
\begin{gathered}
S_{\ell}^{i+k \cdot j+k}=S_{1 \ell}^{i j}, \quad S_{\ell+k}^{i+k \cdot j+k}=S_{2 \ell}^{i j}, \quad S_{\ell}^{i \cdot j+k}=S_{3 \ell}^{i j}, \quad S_{\ell}^{i+k \cdot j}=S_{4 \ell}^{i j}, \\
S_{\ell+k}^{i+k \cdot j}=S_{5 \ell}^{i j}, \quad S_{\ell+k}^{i \cdot j+k}=S_{6 \ell}^{i j}, \quad S_{\ell}^{i j}=S_{7 \ell}^{i j}, \quad S_{\ell+k}^{i j}=S_{8 \ell}^{i j} .
\end{gathered}
$$

ПреДЛОЖЕНИЕ 12.1. Тензор $S_{\gamma}^{\alpha \beta}(J, \varphi)$ mипа $(2,1)$ тогда и только тогда является инвариантным по отношению $\kappa C$-интегрируемой невырожденной 
гамильтоновой системе (1.1), если все компоненты (12.2) зависят только от переменных $J_{i}$ и справедливы уравнения

$$
S_{4}(J)=S_{2}(J)-S_{3}(J), \quad S_{5}=S_{6}=S_{7}=S_{8}=0 .
$$

Компоненты $S_{1}(J), S_{2}(J)$ и $S_{3}(J)$ являются произвольными гладкими функииями от переменных $J_{1}, \ldots, J_{k}$.

ДокАЗАТЕЛЬСТво. Уравнение инвариантности $L_{V} S=0$ для тензора $S_{\gamma}^{\alpha \beta}$ типа $(2,1)$ имеет вид

$$
\left(L_{V} S\right)_{\gamma}^{\alpha \beta}=\dot{S}_{\gamma}^{\alpha \beta}+V_{, \gamma}^{\tau} S_{\tau}^{\alpha \beta}-V_{, \tau}^{\alpha} S_{\gamma}^{\tau \beta}-V_{, \tau}^{\beta} S_{\gamma}^{\alpha \tau}=0
$$

Подставляя формулы (3.4) и (12.2), мы преобразуем систему (12.4) в следуюшую линейную динамическую систему в пространстве тензоров $S_{a}, a=1, \ldots, 8$ :

$$
\begin{gathered}
\dot{S}_{1}=S_{3}+S_{4}-S_{2}, \quad \dot{S}_{2}=S_{5}+S_{6}, \quad \dot{S}_{3}=S_{7}-S_{6}, \\
\dot{S}_{4}=S_{7}-S_{5}, \quad \dot{S}_{5}=S_{8}, \quad \dot{S}_{6}=S_{8}, \quad \dot{S}_{7}=-S_{8}, \quad \dot{S}_{8}=0 .
\end{gathered}
$$

Используя очевидную верхнетреугольную структуру этой системы и основное свойство первых интегралов (2.8), мы получаем явный вид всех решений уравнений (12.5):

$$
\begin{gathered}
S_{1}(t)=-\widetilde{S}_{8}(J) t^{3}+\left(\widetilde{S}_{7}(J)-\widetilde{S}_{5}(J)-\widetilde{S}_{6}(J)\right) t^{2} \\
+\left(\widetilde{S}_{4}(J)+\widetilde{S}_{3}(J)-\widetilde{S}_{2}(J)\right) t+\widetilde{S}_{1}(J), \\
S_{2}(t)=\widetilde{S}_{8}(J) t^{2}+\left(\widetilde{S}_{5}(J)+\widetilde{S}_{6}(J)\right) t+\widetilde{S}_{2}(J), \\
S_{3}(t)=-\widetilde{S}_{8}(J) t^{2}+\left(\widetilde{S}_{7}(J)-\widetilde{S}_{6}(J)\right) t+\widetilde{S}_{3}(J), \\
S_{4}(t)=-\widetilde{S}_{8}(J) t^{2}+\left(\widetilde{S}_{7}(J)-\widetilde{S}_{5}(J)\right) t+\widetilde{S}_{4}(J), \\
S_{5}(t)=\widetilde{S}_{8}(J) t+\widetilde{S}_{5}(J), \quad S_{6}(t)=\widetilde{S}_{8}(J) t+\widetilde{S}_{6}(J), \\
S_{7}(t)=-\widetilde{S}_{8}(J) t+\widetilde{S}_{7}(J), \quad S_{8}(t)=\widetilde{S}_{8}(J),
\end{gathered}
$$

где $\widetilde{S}_{a}(J)$ - произвольные гладкие функции переменных $J_{1}, \ldots, J_{k}$.

$C$-инвариантный тензор $S_{\gamma}^{\alpha \beta}(J, \varphi)$ типа $(2,1)$ является гладким в любой тороидальной области $\mathscr{O}=B_{a} \times \mathbb{T}^{k}$. Поэтому все компоненты $S_{\gamma}^{\alpha \beta}(J, \varphi)$ ограничены по модулю на любом торе $\mathbb{T}^{k}$. Точные решения (12.6) ограничены при всех $t$ тогда и только тогда, если выполнены уравнения (12.3). Поэтому, используя тот факт, что общие траектории интегрируемой невырожденной гамильтоновой системы (1.1) всюду плотны на торах $\mathbb{T}^{k}(2.4)$, мы получаем, что все компоненты $S_{\gamma}^{\alpha \beta}$ зависят только от переменных $J_{1}, \ldots, J_{k}$ и удовлетворяют уравнениям (12.3).

Уравнения (12.3) определяют особые точки динамической системы (12.5). Поэтому тензор $S_{\gamma}^{\alpha \beta}(12.2)$ типа $(2,1)$ является $C$-инвариантным тогда и только тогда, если функции $S_{a}(J)$ зависят только от переменных $J_{1}, \ldots, J_{k}$ и их значения принадлежат особому множеству (12.3) динамической системы (12.5). Прямая проверка доказывает, что уравнения (12.3) влекут тождество

$$
D S(\zeta, \eta)=S(D \zeta, \eta)+S(\zeta, D \eta)
$$

где $D-C$-инвариантный нильпотентный тензор $(2.12),(2.13)$ типа $(1,1)$ и $\zeta, \eta$ - произвольные ковекторы. Тождество (12.7) означает, что тензор $D$ является 
дифференцированием алгебраической структуры (12.1) в кокасательном расслоении $T^{*}\left(M^{n}\right)$.

II. Пусть $\mathscr{L}^{\perp} \subset T^{*}\left(M^{n}\right)$ означает $k$-мерное распределение, порожденное 1 -формами $d J_{1}, \ldots, d J_{k}(2.10)$. Уравнения (12.3) влекут

$$
S\left(\mathscr{L}^{\perp}, \mathscr{L}^{\perp}\right)=0, \quad S\left(\mathscr{L}^{\perp}, T_{x}^{*}\left(M^{n}\right)\right) \subset \mathscr{L}^{\perp}, \quad S\left(T_{x}^{*}\left(M^{n}\right), \mathscr{L}^{\perp}\right) \subset \mathscr{L}^{\perp} .
$$

Поэтому $k$-мерные подпространства $\mathscr{L}_{x}^{\perp}$ являются коммутативными идеалами по отношению к алгебраической структуре (12.1).

Для любой 1-формы $\eta$ мы определяем оператор $S_{\eta}$ по формуле $S_{\eta}(\zeta)=S(\zeta, \eta)$. Рассмотрим эти операторы в базисе $(2.10) e^{i}=d J_{i}, e^{i+k}=d \varphi_{i}$. Формулы (12.8) означают, что операторы $S_{e^{\alpha}}$ имеют вид

$$
S_{e^{i}}=\left(\begin{array}{cc}
0 & Q_{i} \\
0 & 0
\end{array}\right), \quad S_{e^{i+k}}=\left(\begin{array}{cc}
U_{i} & W_{i} \\
0 & V_{i}
\end{array}\right)
$$

где $U_{i}, V_{i}, W_{i}$ и $Q_{i}$ - некоторые $k \times k$ матрицы. Введем следуюшую полиномиальнозначную функцию

$$
P_{S}(\eta, \lambda)=\operatorname{det}\left(S_{\eta}-\lambda\right)
$$

на кокасательном расслоении $T^{*}\left(M^{n}\right)$.

ПРЕДЛОЖЕНИЕ 12.2. Если тензор $S_{\gamma}^{\alpha \beta}$ mипа $(2,1)$ является $C$-инвариантным , то многочлен $P_{S}(\eta, \lambda)$ приводим и является произведением двух многочленов степени $k$. Для любого ковектора $\eta$ и ковектора $\theta \in \mathscr{L}^{\perp}$ справедливь тождества

$$
P_{S}(\eta+\theta, \lambda)=P_{S}(\eta, \lambda), \quad P_{S}(\theta, \lambda)=\lambda^{n}
$$

ДокАЗАТЕЛьство. Ковектор $\eta$ имеет вид

$$
\eta=\rho_{1} e^{1}+\cdots+\rho_{k} e^{k}+\eta_{1} e^{1+k}+\cdots+\eta_{k} e^{2 k}
$$

в базисе (2.10). Используя формулы (12.9), получаем

$$
P_{S}(\eta, \lambda)=\operatorname{det}\left(\eta_{i} U_{i}-\lambda\right) \operatorname{det}\left(\eta_{i} V_{i}-\lambda\right)
$$

Эта формула представляет многочлен (12.10) в виде произведения двух многочленов степени $k$. Формулы (12.11) следуют из (12.12).

III. ТЕОРема 1, ЧАСТЬ 9. Любие две симметрии $U$ и $U_{1}$ C-интегрируемой невърожсднной гамильтоновой системы (1.1), любъе две С-инвариантные 1-формы $\eta$ и $\theta$ июбой $C$-инвариантньй тензор $S_{\gamma}^{\alpha \beta}$ типа $(2,1)$ удовлетворяют уравнениям

$$
S_{\gamma}^{\alpha \beta} \eta_{\alpha} \theta_{\beta}=0, \quad S_{\gamma}^{\alpha \beta} U^{\gamma} \theta_{\beta}=0, \quad L_{U_{1}} L_{U} S=0
$$

2) C-инвариантныи тензор $\left(S_{\theta}\right)_{\beta}^{\alpha}=S_{\beta}^{\alpha \gamma} \theta_{\gamma}$ типа $(1,1)$ и C-инвариантньй тензор $\left(S_{U}\right)^{\alpha \beta}=S_{\gamma}^{\alpha \beta} U^{\gamma}$ типа $(2,0)$ удовлетворяют уравнениям

$$
\begin{gathered}
\operatorname{rank}\left\|S_{\theta}\right\| \leqslant k, \quad S_{\theta}^{2}=0, \quad L_{U} S_{\theta}=0, \\
\operatorname{rank}\left\|S_{U}\right\| \leqslant k, \quad L_{U_{1}} S_{U}=0 .
\end{gathered}
$$


3) Тензор $\widetilde{S}=L_{U} S$ типа $(2,1)$ определяет нильпотентную алгебраическую структуру в кокасательном расслоении $T^{*}\left(M^{n}\right)$ : для произвольных ковекторов $\zeta, \eta, \theta \in T_{x}^{*}\left(M^{n}\right)$ справедливъ уравнения

$$
\widetilde{S}(\widetilde{S}(\zeta, \eta), \theta)=0, \quad \widetilde{S}(\zeta, \widetilde{S}(\eta, \theta))=0 .
$$

4) Многочлен $P_{S}(\eta, \lambda)$ удовлетворяет уравнениям

$$
P_{S}(\eta+\theta, \lambda)=P_{S}(\eta, \lambda), \quad P_{S}(\theta, \lambda)=\lambda^{n}
$$

для любого ковектора $\eta \in T_{x}^{*}\left(M^{n}\right)$ и любой $C$-инвариантной 1-формы $\theta$. Многочлен $P_{S}(\eta, \lambda)$ приводим и является произведением двух многочленов степени $k$.

5) Для любых двух С-инвариантных тензоров $S$ и $Q$ типа $(2,1)$, любой $C$-инвариантной 1-формы $\theta$ и любой симметрии $U$, тензор $P^{\alpha \beta}=S_{\nu}^{\alpha \tau} Q_{\tau}^{\beta \nu}$ типа $(2,0)$ удовлетворяет уравнениям

$$
\operatorname{rank}\|P\| \leqslant k, \quad P^{\alpha \beta} \theta_{\beta}=0, \quad L_{U} P=0 .
$$

6) Для любых $C$-инвариантных тензора $N$ типа $(1,2)$ и тензора $S$ типа $(2,1)$ и любой симметрии $U$ С-инвариантнье тензоры $A_{\beta}^{\alpha}=N_{\tau \nu}^{\alpha} S_{\beta}^{\tau \nu} u$ $B_{\beta}^{\alpha}=N_{\beta \nu}^{\tau} S_{\tau}^{\alpha \nu}$ удовлетворяют уравнениям

$$
\begin{gathered}
\operatorname{rank}\|A\| \leqslant k, \quad \operatorname{rank}\|B\| \leqslant k, \quad A^{2}=B^{2}=A B=B A=0, \\
L_{U} A=L_{U} B=0 .
\end{gathered}
$$

ДоказАтЕльство. 1) Первое уравнение (12.13) следует из первого уравнения (12.8), поскольку любая $C$-инвариантная 1-форма $\theta$ (3.2) принадлежит распределению $\mathscr{L}^{\perp}$. Второе уравнение (12.13) следует из формул (2.15), (3.2) и (12.3) для $C$-инвариантных тензоров $U, \theta$ и $S$.

Применяя предложение 12.1 и форомуы $(2.16),(2.18)$ и (12.4), мы получаем, что ненулевые компоненты тензора $L_{U} S$ типа $(2,1)$ имеют вид

$$
\left(L_{U} S\right)_{\ell}^{i+k \cdot j+k}=U_{, \ell}^{m+k} S_{m+k}^{i+k . j+k}-U_{, m}^{i+k} S_{\ell}^{m \cdot j+k}-U_{, m}^{j+k} S_{\ell}^{i+k \cdot m} .
$$

Поэтому только первая группа компонент $(12.2)$ тензора $\widetilde{S}=L_{U} S$ типа $(2,1)$ является ненулевой. Применяя формулу (12.18) к тензору $L_{U_{1}} \widetilde{S}=L_{U_{1}} L_{U} S$ типа $(2,1)$, мы получаем третье уравнение (12.13).

2) Вследствие формул (2.15), (3.2) и уравнений (12.2), (12.3), все ненулевые компоненты тензоров $S_{\theta}$ и $S_{U}$ имеют вид

$$
\left(S_{\theta}\right)_{j}^{i+k}=S_{j}^{i+k \cdot \ell} \theta_{\ell}, \quad S_{U}^{i+k . j+k}=S_{\ell+k}^{i+k . j+k} U^{\ell+k} .
$$

Отсюда следуют алгебраические уравнения (12.14). Применяя формулы (7.13) и (8.9), мы получаем уравнения $L_{U} S_{\theta}=0$ и $L_{U_{1}} S_{U}=0$.

3) Мы доказали, что все компоненты тензора $\widetilde{S}=L_{U} S$ типа $(2,1)$ равны нулю, за исключением компонент $\left(L_{U} S\right)_{\ell}^{i+k \cdot j+k}$. Этот факт означает, что тензор $\widetilde{S}$ типа $(2,1)$ удовлетворяет уравнениям

$$
\widetilde{S}\left(T_{x}^{*}\left(M^{n}\right), T_{x}^{*}\left(M^{n}\right)\right) \subset \mathscr{L}_{x}^{\perp}, \quad \widetilde{S}\left(\mathscr{L}_{x}^{\perp}, T_{x}^{*}\left(M^{n}\right)\right)=\widetilde{S}\left(T_{x}^{*}\left(M^{n}\right), \mathscr{L}_{x}^{\perp}\right)=0 .
$$


Отсюда следуют уравнения (12.15).

4) Утверждение (4) следует из предложения 12.2 , поскольку все $C$-инвариантные 1-формы $\theta$ принадлежат распределению $\mathscr{L}^{\perp}$ вследствие (3.2).

$5)$ Используя формулы (12.2) и (12.3) для любого $C$-инвариантного тензора типа $(2,1)$, мы получаем, что все ненулевые компоненты тензора $P$ типа $(2,0)$ имеют вид

$$
P^{i+k \cdot j+k}=S_{m}^{i+k \cdot \ell} Q_{\ell}^{j+k \cdot m}+S_{m+k}^{i+k \cdot \ell+k} Q_{\ell+k}^{j+k \cdot m+k} .
$$

Отсюда следует первое неравенство (12.16). Второе уравнение (12.16) следует из формулы (3.2) для $C$-инвариантной 1-формы $\theta$ и (12.19). Применяя формулу Лейбница, мы находим

$$
\left(L_{U} P\right)^{\alpha \beta}=\left(L_{U} S\right)_{\nu}^{\alpha \tau} Q_{\tau}^{\beta \nu}+S_{\nu}^{\alpha \tau}\left(L_{U} Q\right)_{\tau}^{\beta \nu} .
$$

Используя формулы (12.18) для ненулевых компонент тензоров $L_{U} S$ и $L_{U} Q$ типа $(2,1)$ и формулы $(12.3)$, мы получаем, что обе суммы в (12.20) равны нулю. Поэтому $L_{U} P=0$ для любой симметрии $U$.

6) Используя формулы (9.4) и (12.3) для $C$-инвариантных тензора $N$ типа $(1,2)$ и тензора $S$ типа $(2,1)$, мы получаем, что все ненулевые компоненты тензоров $A_{\beta}^{\alpha}=$ $N_{\tau \nu}^{\alpha} S_{\beta}^{\tau \nu}$ и $B_{\beta}^{\alpha}=N_{\beta \nu}^{\tau} S_{\tau}^{\alpha \nu}$ типа $(1,1)$ имеют вид

$$
\begin{aligned}
& A_{j}^{i+k}=N_{\ell+k . m}^{i+k} S_{j}^{\ell+k . m}+N_{\ell \cdot m+k}^{i+k} S_{j}^{\ell . m+k}, \\
& B_{j}^{i+k}=N_{j . m}^{\ell} S_{\ell}^{i+k . m}+N_{j . m+k}^{\ell+k} S_{\ell+k}^{i+k . m+k} .
\end{aligned}
$$

Поэтому тензоры $A$ и $B$ имеют блочную структуру (8.10). Отсюда следуют алгебраические уравнения (12.17). Уравнения $L_{U} A=L_{U} B=0$ следуют из равенства $(8.9)$ и формул $(2.15)$ и (12.21).

\section{$\S 13 . \quad A-B-C$-когомологии динамических систем}

I. Напомним конструкцию $A-B-C$-когомологий динамических систем, предложенную в работах [2], [4], [5]. Пусть $\Lambda_{V}^{m}$ - линейное пространство гладких дифференциальных $m$-форм на многообразии $M^{n}$, которые инвариантыы по отношению к динамической системе $V$

$$
\dot{x}^{i}=V^{i}\left(x^{1}, \ldots, x^{n}\right)
$$

на $M^{n}$. Оператор $i_{V}$ внутреннего умножения и оператор $d$ внешнего дифференцирования действуют на $V$-инвариантных дифференциальных формах. Любая $V$-инвариантная $m$-форма $\omega_{m}$ аннулируется производной Ли

$$
L_{V}=i_{V} \circ d+d_{\circ} i_{V}, \quad L_{V} \omega_{m}=0 .
$$

Поэтому два оператора $i_{V}$ и $d$ удовлетворяют уравнениям

$$
i_{V}^{2}=0, \quad d^{2}=0, \quad i_{V} \circ d=-d_{\circ} i_{V}
$$

на линейном пространстве $V$-инвариантных дифференциальных форм. Вследствие уравнений (13.2), оператор $d_{V}=i_{V} \circ d=-d_{\circ} i_{V}$ удовлетворяет уравнениям

$$
d_{V}^{2}=0, \quad d_{V} \circ i_{V}=i_{V} \circ d_{V}=0, \quad d_{V} \circ d=d \circ d_{V}=0 .
$$


Линейные подпространства $\Lambda_{V}^{m} V$-инвариантных дифференциальных $m$-форм с тремя операторами $i_{V}, d$ и $d_{V}=i_{V}$ od образуют $A-, B-$, и $C$-комплексы соответственно:

$$
\begin{aligned}
& A: 0 \longleftarrow \Lambda_{V}^{0} \stackrel{i_{V}}{\longleftarrow} \Lambda_{V}^{1} \stackrel{i_{V}}{\longleftarrow} \ldots \stackrel{i_{V}}{\longleftarrow} \Lambda_{V}^{n-1} \stackrel{i_{V}}{\longleftarrow} \Lambda_{V}^{n} \longleftarrow 0 \\
& B: 0 \longrightarrow \Lambda_{V}^{0} \stackrel{d}{\longrightarrow} \Lambda_{V}^{1} \stackrel{d}{\longrightarrow} \ldots \stackrel{d}{\longrightarrow} \Lambda_{V}^{n-1} \stackrel{d}{\longrightarrow} \Lambda_{V}^{n} \longrightarrow 0 \\
& C: \quad \stackrel{d_{V}}{\longrightarrow} \Lambda_{V}^{m} \stackrel{d_{V}}{\longrightarrow} \Lambda_{V}^{m} \stackrel{d_{V}}{\longrightarrow} \Lambda_{V}^{m} \stackrel{d_{V}}{\longrightarrow} .
\end{aligned}
$$

II. Линейные пространства $H_{A}^{*}\left(V, M^{n}\right), H_{B}^{*}\left(V, M^{n}\right)$ и $H_{C}^{*}\left(V, M^{n}\right)$ являются когомологиями по отношению к операторам $i_{V}, d$ и $d_{V}$ соответственно:

$$
\begin{aligned}
H_{A}^{*}\left(V, M^{n}\right)= & \operatorname{Ker} i_{V} / \operatorname{Im} i_{V}, \quad H_{B}^{*}\left(V, M^{n}\right)=\operatorname{Ker} d / \operatorname{Im} d \\
& H_{C}^{*}\left(V, M^{n}\right)=\operatorname{Ker} d_{V} / \operatorname{Im} d_{V} .
\end{aligned}
$$

Кольцевые структуры в когомологиях (13.4) индуцируются внешним произведением $V$-инвариантных дифференциальных форм. Факторпространства (13.4) наследуют кольцевую структуру, определенную внешним произведением $V$-инвариантных дифференциальных форм, вследствие того, что операторы $i_{V}$ и $d$ являются косыми дифференцированиями [15] и оператор $d_{V}=i_{V}$ о $d$ является дифференцированием:

$$
d_{V}(\omega \wedge \eta)=d_{V} \omega \wedge \eta+\omega \wedge d_{V} \eta
$$

Здесь $\omega$ и $\eta$ - произвольные $V$-инвариантные дифференциальные формы.

Для любой постоянной $c$ оператор $d_{c}=d+c i_{V}$ удовлетворяет уравнению $d_{c}^{2}=0$ на $V$-инвариантных дифференциальных формах. Поэтому можно определить когомологии по отношению к оператору $d_{c}$. $A$-когомологии $H_{A}^{*}\left(V, M^{n}\right)$ образуют дифференциальный комплекс по отношению к оператору $d$. $B$-когомологии $H_{B}^{*}\left(V, M^{n}\right)$ образуют дифференциальный комплекс по отношению к оператору $i_{V}$. $C$-когомологии $H_{C}^{*}\left(V, M^{n}\right)$ образуют два дифференциальных комплекса по отношению к операторам $i_{V}$ и $d$. Когомологии этих дифференциальных комплексов определяют дополнительные инварианты динамической системы $V$.

III. Рассмотрим динамическую систему $V(2.6)$ :

$$
\dot{J}_{i}=0, \quad \dot{\varphi}_{i}=J_{i}
$$

в тороидальной области $\mathscr{O}=B_{a} \times \mathbb{T}^{k}$, где $B_{a}-k$-мерньй шар, и $J_{i} \in B_{a}, \varphi_{i} \in \mathbb{T}^{k}$. Система (2.6) является канонической формой [3] всех $C$-интегрируемых гамильтоновых систем, невырожденных в смысле Пуанкаре (2.3). В $\S 3,6$ и 10 мы показали, что $C$-инвариантные 1-, 2- и 3-формы имеют канонический вид

$$
\begin{aligned}
& \omega_{1}=\theta_{i}(J) d J_{i} \\
& \omega_{2}=a_{i \ell}(J) d J_{i} \wedge d J_{\ell}+b_{i \ell}(J) d J_{i} \wedge d \varphi_{\ell} \\
& \omega_{3}=b_{i \ell m}(J) d J_{i} \wedge d J_{\ell} \wedge d \varphi_{m}+c_{i \ell m}(J) d J_{i} \wedge d J_{\ell} \wedge d J_{m}
\end{aligned}
$$

Здесь коэффициенты $a_{i \ell}(J)$ и $c_{i \ell m}(J)$ являются знакопеременньми и коэффициенты $b_{i \ell}(J)$ и $b_{i \ell m}(J)$ удовлетворяют уравнениям $b_{i \ell}(J)=b_{\ell i}(J), b_{i \ell m}(J)+b_{\ell m i}(J)+$ $b_{m i \ell}(J)=0$.

(А) Для $C$-инвариантных дифференциальных форм (13.5)-(13.7), мы имеем

$$
i_{V} \omega_{1}=0, \quad i_{V} \omega_{2}=-b_{i \ell}(J) J_{\ell} d J_{i}, \quad i_{V} \omega_{3}=b_{i \ell m}(J) J_{m} d J_{i} \wedge d J_{\ell} .
$$


Отсюда мы получаем $H_{A}^{1}(V, \mathscr{O})=0, H_{A}^{2}(V, \mathscr{O})=\mathbb{R}^{\infty}$. Векторы из групшы когомологий $H_{A}^{2}(V, \mathscr{O})$ представляются 2-формами

$$
\omega_{2 a}=b_{i \ell}(J) d J_{i} \wedge d \varphi_{\ell},
$$

где $b_{i \ell}(J)=b_{\ell i}(J)$ и $b_{i \ell}(J) J_{\ell}=0$.

(В) Любая $C$-инвариантная 1-форма $\theta$ имеет вид (13.5). Поэтому, применяя лемму Пуанкаре к замкнутой 1-форме (13.5), мы получаем $\theta=d F(J)$. Поэтому $H_{B}^{1}(V, \mathscr{O})=0$. В предложении 6.1 мы доказали, что любая $C$-инвариантная замкнутая 2-форма $\omega_{2}$ имеет вид (6.3). Поэтому векторы из когомологической группы $H_{B}^{2}(V, \mathscr{O})$ представляются замкнутыми 2-формами

$$
\omega_{2 b}=\frac{\partial^{2} B(J)}{\partial J_{i} \partial J_{\ell}} d J_{i} \wedge d \varphi_{\ell}
$$

где $B(J)$ - произвольная гладкая функция. Когомологические классы в группе $H_{B}^{2}(V, \mathscr{O})$ определяются классами гладких функций $B(J)$ по модулю линейных функций $c_{1} J_{1}+\cdots+c_{k} J_{k}$. Поэтому группа когомологий $H_{B}^{2}(V, \mathscr{O})$ является бесконечномерной.

(C) Уравнение (13.6) и (13.8) приводят к формулам

$$
d_{V} \omega_{2}=-d i_{V} \omega_{2}=d\left(b_{i \ell}(J) J_{\ell}\right) \wedge d J_{i} .
$$

Поэтому группа когомологий $H_{C}^{2}\left(V, M^{n}\right)$ бесконечномерна. Векторы этой группы представляются 2-формами

$$
\omega_{2 c}=a_{i \ell}(J) d J_{i} \wedge d J_{\ell}+b_{i \ell}(J) d J_{i} \wedge d \varphi_{\ell}, \quad b_{i \ell}(J) J_{\ell}=\frac{\partial C(J)}{\partial J_{i}},
$$

где $C(J)$ - произвольная гладкая функция и $b_{i \ell}(J)=b_{\ell i}(J)$.

Формулы (13.9)-(13.11) показывают, что три группы когомологий

$$
H_{A}^{2}(V, \mathscr{O}), \quad H_{B}^{2}(V, \mathscr{O}), \quad H_{C}^{2}(V, \mathscr{O})
$$

отличаются друг от друга и что $B$-когомологическая группа $H_{B}^{2}(V, \mathscr{O})$ является "наименьшей" из них.

$B$-когомологии являются наиболее важными из $A-B$ - $C$-типов вследствие их связей с классическими когомологиями де Рама [20]. B-когомологии динамических систем имеют аналоги для дискретных динамических систем, которые определяются гладкими отображениями $f: M^{n} \rightarrow M^{n}$ многообразия $M^{n}$ в себя.

\section{§ 14. $B$-когомологии динамических систем и гладких отображений}

I. Существует естественньй кольцевой гомоморфизм

$$
\alpha: H_{B}^{*}\left(V, M^{n}\right) \rightarrow H^{*}\left(M^{n}\right),
$$

который отображает класс $B$-когомологий $V$-инвариантной замкнутой $q$-формы $\omega_{q}$ в соответствующий класс когомологий де Рама [20] общих замкнутых $q$-форм.

Для любой постоянной $c \neq 0$ имеется изоморфизм

$$
H_{B}^{*}\left(c V, M^{n}\right)=H_{B}^{*}\left(V, M^{n}\right) .
$$


При $c=0$ когомологии $H_{B}^{*}\left(0, M^{n}\right)$ изоморфны когомологиям де Рама $H^{*}\left(M^{n}\right)$.

Гомоморфизм $\alpha$ имеет обратный и поэтому является изоморфизмом для следующих динамических систем.

1) Предположим, что все траектории динамической системы (13.1) замкнуты и имеют одинаковьй период $T$. Обозначим через

$$
\psi_{\tau}: M^{n} \rightarrow M^{n}, \quad \psi_{T}=\mathrm{id}
$$

соответствующее действие окружности $S^{1}$. Для любой замкнутой $q$-формы $\omega_{q}$ мы определяем $q$-форму

$$
\alpha^{-1} \omega_{q}=\frac{1}{T} \int_{0}^{T} \psi_{\tau}^{*}\left(\omega_{q}\right) d \tau
$$

Очевидно, что $q$-форма $\alpha^{-1} \omega_{q}$ замкнута и инвариантна по отношению ко всем диффеоморфизмам (14.1) и что $\alpha^{-1}$ о $\alpha=\mathrm{id}$. Эта $q$-форма $\alpha^{-1} \omega_{q}$ принадлежит тому же классу когомологий де Рама в $H^{q}\left(M^{n}\right)$, что и замкнутая $q$-форма $\omega_{q}$, потому что $q$-формы $\psi_{\tau}^{*}\left(\omega_{q}\right)$ гомотопны $\omega_{q}$ при всех $\tau$. Поэтому $\alpha \circ \alpha^{-1}=\mathrm{id}$ в $H^{q}\left(M^{n}\right)$. Следовательно отображение $\alpha$ является изоморфизмом.

2) Пусть $M^{n}=X^{n-k} \times \mathbb{T}^{k}$, где $X^{n-k}$-гладкое $(n-k)$-мерное многообразие с атласом локальных координат $x^{1}, \ldots, x^{n}$ и $\mathbb{T}^{k}-k$-мерный тор с угловыми координатами $\varphi_{1}, \ldots, \varphi_{k}$.

Рассмотрим динамическую систему

$$
\dot{x}^{i}=0, \quad \dot{\varphi}_{j}=b_{j}
$$

на многообразии $M^{n}$. Здесь $b_{j}$ - произвольные рационально независимые постоянные. Динамическая система (14.2) определяет следуюшую группу диффеоморфизмов

$$
\phi_{t}:\left(x^{i}, \varphi_{j}\right) \rightarrow\left(x^{i}, \varphi_{j}+t b_{j}\right)
$$

многообразия $M^{n}$ в себя.

Пусть $f(x, \varphi)$ - произвольная гладкая функция на $M^{n}$. Применяя эргодическую теорему [21] для подсистемы (14.2) на торе $\mathbb{T}^{k}$, получаем равенство

$$
\overline{f(x)}=\lim _{T \rightarrow \infty} \frac{1}{T} \int_{0}^{T} f\left(x^{i}, \varphi_{j}+t b_{j}\right) d t=\frac{1}{(2 \pi)^{k}} \int_{\mathbb{T}^{k}} f(x, \varphi) d \varphi_{1} \wedge \cdots \wedge d \varphi_{k} .
$$

Пусть $\omega_{q}-$ произвольная замкнутая $q$-форма

$$
\omega_{q}=\sum_{\ell+m=q} \sum_{i, j} a_{i . j}(x, \varphi) d x^{i_{1}} \wedge \cdots \wedge d x^{i_{\ell}} \wedge d \varphi_{j_{1}} \wedge \cdots \wedge d \varphi_{j_{m}}
$$

на многообразии $M^{n}$. Для всех диффеоморфизмов $\phi_{t}$ (14.3), дифференциалы $d \phi_{t}$ являются тождественньми отображениями касательных пространств $T_{(x, \varphi)}\left(X^{n-k} \times \mathbb{T}^{k}\right)$. Используя этот факт, выводим равенство

$$
\lim _{T \rightarrow \infty} \frac{1}{T} \int_{0}^{T} \phi_{t}^{*}\left(\omega_{q}\right) d t=\sum_{\ell+m=q} \sum_{i, j} \overline{a_{i . j}(x)} d x^{i_{1}} \wedge \cdots \wedge d x^{i_{\ell}} \wedge d \varphi_{j_{1}} \wedge \cdots \wedge d \varphi_{j_{m}} .
$$

Определим $q$-форму

$$
\alpha^{-1} \omega_{q}=\bar{\omega}_{q}=\lim _{T \rightarrow \infty} \frac{1}{T} \int_{0}^{T} \phi_{t}^{*}\left(\omega_{q}\right) d t
$$


Очевидно, что $q$-форма $\omega_{q}$ является замкнутой и инвариантной по отношению к динамической системе (15.2). Поэтому $\bar{\omega}_{q} \in H_{B}^{q}\left(V, M^{n}\right)$. Очевидно, что $\alpha^{-1} \circ \alpha=$ id.

$V$-инвариантная замкнутая $q$-форма $\bar{\omega}_{q}$ принадлежит тому же классу когомологий де Рама в $H^{q}\left(M^{n}\right)$, что и замкнутая $q$-форма $\omega_{q}$, потому что $q$-формы $\phi_{t}^{*}\left(\omega_{q}\right)$ гомотопны $\omega_{q}$ при всех $t$. Поэтом у $\alpha \circ \alpha^{-1}=\mathrm{id} \mathrm{в} H^{q}\left(M^{n}\right)$. Следовательно отображение $\alpha$ является изоморфизмом. Таким образом, мы доказали изоморфизм двух когомологий

$$
\alpha: H_{B}^{*}\left(V, M^{n}\right)=H^{*}\left(M^{n}\right)
$$

для динамической системы (14.2) на многообразии $M^{n}=X^{n-k} \times \mathbb{T}^{k}$.

Классический гармонический осциллятор

$$
\dot{p}_{i}=-a_{i} q_{i}, \quad \dot{q}_{i}=\frac{1}{m_{i}} p_{i}
$$

является примером системы (14.2). Поэтому для гармонического осциллятора кольцо $B$-когомологий

$$
H_{B}^{*}\left(V, \mathbb{R}^{2 k}\right)=H^{*}\left(\mathbb{R}^{2 k}\right)=H^{0}\left(\mathbb{R}^{2 k}\right)=\mathbb{R}^{1}
$$

изоморфно кольцу вешественных чисел $\mathbb{R}^{1}$.

II. Предположим, что динамическая система (13.1) является общей неинтегрируемой гамильтоновой системой. Тогда $V^{i}=P_{1}^{i j} \partial H(x) / \partial x^{j}$, где $P_{1}$ - невырожденная пуассонова структура на $M^{2 k}$. Соответствующие $B$-когомологии изомор ф̆ны сумме

$$
H_{B}^{*}\left(V, M^{2 k}\right)=\mathbb{R}[u] / u^{k+1} \mathbb{R}[u]+H_{B}^{2 k}\left(V, M^{2 k}\right)
$$

факторкольца $\mathbb{R}[u] / u^{k+1} \mathbb{R}[u]$ многочленов от одной переменной $u$ и бесконечномерной группы $H_{B}^{2 k}\left(V, M^{2 k}\right)$ с тривиальным законом умножения. Образуюшая $u \in H_{B}^{2}\left(V, M^{2 k}\right)$ соответствует $V$-инвариантной симплектической структуре $\omega_{1}=P_{1}^{-1}$. Линейно независимые элементы бесконечномерной группы $H_{B}^{2 k}\left(V, M^{2 k}\right)$ представляются $V$-инвариантными замкнутыми $2 k$-формами

$$
\omega_{F}=F(H) \omega_{1} \wedge \cdots \wedge \omega_{1} .
$$

Внешнее произведение (14.6) содержит $k$ сомножителей $\omega_{1}, F(H)$ - произвольная гладкая функция одной переменной и $H(x)$ - гамильтониан системы $V$.

Изоморфизм (14.4) для интегрируемой динамической системы (14.2) и изоморфизм (14.5) для общей неинтегрируемой гамильтоновой системы $V$ показывают, что кольцо когомологий $H_{B}^{*}\left(V, M^{n}\right)$ является новым инвариантом, который характеризует одновременно топологические свойства многообразия $M^{n}$ и глобальные свойства динамической системы $V$ на $M^{n}$.

III. Рассмотрим $C$-интегрируемую невырожденную гамильтонову систему (2.1) в тороидальной области $\mathscr{O}=B_{a} \times \mathbb{T}^{k}$. Гамильтонова система (2.1) имеет вид (2.6) в тороидальных координатах $J_{\ell}(I)=\partial H(I) / \partial I_{\ell}, \varphi_{\ell}$.

Теорема 2. Первъе пять групп В-когомологий для $C$-интегрируемой невыродденной гамильтоновой системы $V(2.1)$ в тороидальной области $\mathscr{O}$ имеют вид

$$
\begin{gathered}
H_{B}^{0}(V, \mathscr{O})=\mathbb{R}^{1}, \quad H_{B}^{1}(V, \mathscr{O})=0, \quad H_{B}^{2}(V, \mathscr{O})=\mathbb{R}^{\infty}, \\
H_{B}^{3}(V, \mathscr{O})=0, \quad H_{B}^{4}(V, \mathscr{O})=\mathbb{R}^{\infty} .
\end{gathered}
$$


ДокАЗАТЕльство. 1) Предложение 3.1 утверждает, что любая $C$-инвариантная 1-форма $\theta$ имеет канонический вид (3.2). Применяя лемму Пуанкаре, мы получаем $\theta=d F(J)$, где $F(J)$ - некоторый первый интеграл системы (2.6). Поэтому $H_{B}^{1}(V, \mathscr{O})=0$.

2) Предложение 6.1 утверждает, что любая $C$-инвариантная замкнутая 2-форма $\omega_{2}$ имеет канонический вид (6.3). Вследствие (3.2), любая $C$-инвариантная точная 2-форма имеет вид

$$
d \theta=d \theta_{\ell}(J) \wedge d J_{\ell}
$$

Поэтому все $C$-инвариантные замкнутые 2-формы $\omega_{2}(6.3)$ с нелинейными функциями $B(J)$ не является точньми. Отсюда мы получаем $H_{B}^{2}(V, \mathscr{O})=\mathbb{R}^{\infty}$.

3) Предложение 10.2 утверждает, что любая $C$-инвариантная замкнутая 3 -форма $\omega_{3}$ является точной в тороидальной области $\mathscr{O}$ и имеет вид $\omega_{3}=d \widetilde{\omega}_{2}$, где 2 -форма $\widetilde{\omega}_{2}(10.10) C$-инвариантна. Поэтому $H_{B}^{3}(V, \mathscr{O})=0$.

4) Пусть $\omega_{i}-C$-инвариантные замкнутые 2-формы (6.3)

$$
\omega_{i}=d\left(\frac{\partial B_{i}(J)}{\partial J_{\ell}}\right) \wedge d \varphi_{\ell}+d f_{\ell}(J) \wedge d J_{\ell},
$$

отвечающие некоторым гладким функциям $B_{i}(J)$. Дифференциальные 4-формы

$$
\omega_{4}=\sum_{i, j=1}^{N} c_{i j} \omega_{i} \wedge \omega_{j}
$$

являются $C$-инвариантными и замкнутыми при произвольных постоянных $c_{i j} . \mathrm{Ka-}$ ноническая форма (10.6)

$$
\omega_{3}=b_{i \ell m}(J) d J_{i} \wedge d J_{\ell} \wedge d \varphi_{m}+c_{i \ell m}(J) d J_{i} \wedge d J_{\ell} \wedge d J_{m}
$$

для любой $C$-инвариантной 3 -формы $\omega_{3}$ доказывает, что равенство $\omega_{4}=d \omega_{3}$ невозможно, если функции $B_{j}(J)$ в $(14.7)$ и постоянные $c_{i j}$ находятся в общем положении. Поэтому группа когомологий $H_{B}^{4}(V, \mathscr{O})$ бесконечномерна.

Применяя методы данной работы, можно доказать, что для любой интегрируемой динамической системы $V(2.1)$, невырожденной в смысле Пуанкаре (2.3), все нечетные когомологии $H_{B}^{2 \ell+1}(V, \mathscr{O})$ равны нулю и все четные когомологии $H_{B}^{2 \ell}(V, \mathscr{O})$ бесконечномерны при $\ell \leqslant k$.

IV. Метод “тороидальных перестроек” [3] определяет гладкое продолжение любой $C$-инвариантной замкнутой 2-формы $\omega_{i}(14.7)$ на все многообразие $M^{2 k}$. Поэтому вторая и четвертая групшы когомологий $H^{2}\left(V, M^{2 k}\right)$ и $H^{4}\left(V, M^{2 k}\right)$ являются бесконечномерными. Таким образом, мы приходим к следующему результату.

СлЕДСТВИЕ 14.1. Бесконечномерность групп когомологий $H_{B}^{2}\left(V, M^{2 k}\right) u$ $H_{B}^{4}\left(V, M^{2 k}\right)$ является необходимым условием для невырожсденной $C$-интегрируемости динамической системы $V$ на многообразии $M^{2 k}$.

$\mathrm{V} . \mathrm{B} \oint 13$ мы рассмотрели $A-B-C$-когомологии динамических систем. Среди них только $B$-когомологии имеют аналоги для гладких отображений. Пусть $f-$ некоторое гладкое отображение многообразия $M^{n}$ в себя. Обозначим через $\Lambda_{f}^{m}$ пространство $f$-инвариантных $m$-форм $\omega_{k}: f^{*} \omega_{m}=\omega_{m}$. Рассмотрим комплекс $f$-инвариантных дифференциальных форм

$$
0 \longrightarrow \Lambda_{f}^{0} \stackrel{d}{\longrightarrow} \Lambda_{f}^{1} \stackrel{d}{\longrightarrow} \ldots \stackrel{d}{\longrightarrow} \Lambda_{f}^{n-1} \stackrel{d}{\longrightarrow} \Lambda_{f}^{n} \longrightarrow 0
$$


Определим $B$-когомологии гладкого отображения $f$ формулой

$$
H_{B}^{*}\left(f, M^{n}\right)=\operatorname{Ker} d / \operatorname{Im} d .
$$

Когомологии $H_{B}^{*}\left(f, M^{n}\right)$ имеют кольцевую структуру, которая индуцируется внешним произведением $f$-инвариантных дифференциальных форм.

Если диффеоморфизм $f_{\tau}$ является сдвигом на время $\tau$ вдоль траекторий некоторой динамической системы $V$, то определен кольцевой гомоморфизм $H_{B}^{*}\left(V, M^{n}\right) \rightarrow$ $H_{B}^{*}\left(f_{\tau}, M^{n}\right)$. Поэтому когомологии $H_{B}^{*}\left(f_{\tau}, M^{n}\right)$ при $\tau \rightarrow 0$ являются дискретизациями когомологий $H_{B}^{*}\left(V, M^{n}\right)$.

Сушествует естественный гомоморфизм $\alpha: H_{B}^{*}\left(f, M^{n}\right) \rightarrow H^{*}\left(M^{n}\right)$, который преобразует класс когомологий $f$-инвариантных $q$-форм в соответствующий класс когомологий де Рама [20] общих замкнутых $q$-форм.

Обозначим $S_{f} M^{n}$ надстройку [22] многообразия $M^{n}$, соответствующую некоторому диффеоморфизму $f: M^{n} \rightarrow M^{n}$. Пусть $t \in[0,1]$ - "вертикальная" координата на $S_{f} M^{n}$, определяюшая векторное поле $V=\partial / \partial t$. Отображение $f$ индуцирует диффеоморфизм $f_{1}$ надстройки $S_{f} M^{n}: f_{1}(x, t)=(f(x), t)$. Очевидно, что диффеоморфизм $f_{1}$ является сдвигом на 1 вдоль траекторий динамической системы $V=\partial / \partial t$ на $S_{f} M^{n}$.

Любая $f$-инвариантная $m$-форма $\omega_{m}$ на $M^{n}$ индуцирует $V$-инвариантную $m$-форму $E \omega_{m}$ на $S_{f} M^{n}$. Эта $m$-форма $E \omega_{m}$ определяется вложениями $E_{t}: M^{n} \rightarrow S_{f} M^{n}, E_{t}(x)=(x, t)$ и удовлетворяет уравнениям $E_{t}^{*}\left(E \omega_{m}\right)=\omega_{m}$, $i_{V} E \omega_{m}=0$.

ПРЕДЛОЖЕНИЕ 14.1. Для любого диффеоморфизма $f: M^{n} \rightarrow M^{n}$ следующие два кольца когомологий изоморфнь:

$$
H_{B}^{*}\left(V, S_{f} M^{n}\right)=H_{B}^{*}\left(f, M^{n}\right) \otimes H^{*}\left(S^{1}\right) .
$$

Здесь $H^{*}\left(S^{1}\right)=\mathbb{R}^{1} \oplus \mathbb{R}^{1}$ - кольцо когомологий окружности $S^{1}$.

Доказательство следует из того факта, что любая $V$-инвариантная $(m+1)$-форма $\eta_{m+1}$ на $S_{f} M^{n}$ имеет вид $\eta_{m+1}=d t \wedge E \omega_{m}+E \omega_{m+1}$, где $\omega_{m}$ и $\omega_{m+1}-$ некоторые $f$-инвариантные формы на $M^{n}$.

Пусть многообразие $M^{2 k}$ является тороидальной областью $\mathscr{O}=B_{a} \times \mathbb{T}^{k}$, где $B_{a} \subset \mathbb{R}^{k}$ - шар (2.2). Определим диффеоморфизм $f: \mathscr{O} \rightarrow \mathscr{O}$ формулой

$$
f\left(I_{j}, \varphi_{j}\right)=\left(I_{j}, \varphi_{j}+\alpha_{j}(I)\right), \quad j=1, \ldots, k,
$$

где $\alpha_{j}(I)$ - произвольные гладкие функции.

ПРЕДЛОЖЕНИЕ 14.2. Предположим, ито диффеоморфизм $f(14.8)$ удовлетворяет условию невырожденности

$$
\operatorname{det}\left\|\frac{\partial \alpha_{\ell}(I)}{\partial I_{j}}\right\| \neq 0, \quad \ell, j=1, \ldots, k,
$$

и что отображсение $\left(I_{1}, \ldots, I_{k}\right) \rightarrow\left(\alpha_{1}(I), \ldots, \alpha_{k}(I)\right)$ является взаимно однозначным. Тогда первъе пять групп В-когомологий имеют вид

$$
\begin{gathered}
H_{B}^{0}(f, \mathscr{O})=\mathbb{R}^{1}, \quad H_{B}^{1}(f, \mathscr{O})=0, \quad H_{B}^{2}(f, \mathscr{O})=\mathbb{R}^{\infty}, \\
H_{B}^{3}(f, \mathscr{O})=0, \quad H_{B}^{4}(f, \mathscr{O})=\mathbb{R}^{\infty} .
\end{gathered}
$$

Доказательство основано на явном виде канонических форм для $f$-инвариантных дифференциальных форм в локальных координатах $\varphi_{j}, J_{\ell}=\alpha_{\ell}(I)$ и аналогично доказательству теоремы 2. 


\section{$\S 15$. Заключительные замечания о свойствах $C$-инвариантных тензоров}

(i) Симметрии $U C$-интегрируемых невырожденных гамильтоновых систем (2.1) на симплектических многообразиях $M^{2 k}$, или $C$-инвариантные $(1,0)$ тензоры, изучались в работе [5]. Теорема о симметриях [5] утверждает, что Ли алгебра $\mathcal{S}$ симметрий интегрируемой по Лиувиллю гамильтоновой системы (2.1) является абелевой тогда и только тогда, если все инвариантные подмногообразия этой системы компактны и система является невырожденной в смысле Пуанкаре.

(ii) $C$-инвариантные дифференциальные 1-формы $\theta$ или $(0,1)$ тензоры удовлетворяют необходимьм условиям

$$
\operatorname{rank} d \theta \leqslant k, \quad \theta(U)=0, \quad L_{U} \theta=0, \quad i_{U} d \theta=0,
$$

где $U$ - произвольная симметрия. Здесь $L_{U}$ означает производную Ли по отношению к симметрии $U$.

(iii) Любой $C$-инвариантный тензор $T_{m}^{\ell}$ типа $(\ell, m)$ для $\ell+m=2$ удовлетворяет соотношению

$$
\operatorname{rank}\left\|L_{U} T_{m}^{\ell}\right\| \leqslant k
$$

При $\ell+m \leqslant 3$, справедливо уравнение

$$
L_{U_{1}} L_{U} T_{m}^{\ell}=0
$$

Здесь $U$ и $U_{1}$ - произвольные симметрии $C$-интегрируемой невырожденной системы (2.1).

(iv) Характеристический многочлен $P(\lambda, x)=\operatorname{det}(A(x)-\lambda)$ любого $C$-инвариантного тензора $A(x)$ типа $(1,1)$ является полньм квадратом: $P(\lambda, x)=Q^{2}(\lambda, x)$. Поэтому каждое собственное значение оператора $A(x)$ имеет четную кратность. Уравнение $\left(L_{U} A\right)^{2}=0$ справедливо для любой симметрии $U$.

(v) Любой $C$-инвариантный тензор $h_{\alpha \beta}$ типа $(0,2)$ удовлетворяет уравнению $h\left(U, U_{1}\right)=0$ для произвольных симметрий $U$ и $U_{1}$. Поэтому любая $C$-инвариантная метрика $g_{\alpha \beta}=g_{\beta \alpha}$ является неопределенной. Числа ее положительных и отрицательных квадратов $n_{+}$и $n_{-}$удовлетворяют неравенствам $n_{+} \leqslant k, n_{-} \leqslant k$. Любая $C$-инвариантная метрика $g_{\alpha \beta}$ при $n=2 k=4 \ell+2$ является вырожденной всюду на многообразии $M^{2 k}$.

(vi) Полная классификация $C$-инвариантных замкнутых дифференциальных 2-форм была получена в работах [1], [3]. Эта классификация доказывает, что общие $C$-инвариантњые пуассоновы структуры являются несовместимыми в смысле Магри и что совместимые $C$-инвариантные пуассоновы структуры являются неустойчивьми.

(vii) Любой $C$-инвариантный тензор $P^{\alpha \beta}$ типа $(2,0)$ удовлетворяет уравнению $P\left(\theta, \theta_{1}\right)=0$, где $\theta$ и $\theta_{1}$ - любые $C$-инвариантные 1 -формы. Для любого кососимметричного $C$-инвариантного тензора $P$ типа $(2,0)$ и любой симметрии $U$, производная Ли $L_{U} P$ является пуассоновой структурой. Если $P$ является пуассоновой структурой, то $P$ и $L_{U} P$ совместимы в смысле Магри. Для любых двух $C$-инвариантных кососимметричных тензоров $P$ и $P_{1}$ типа $(2,0)$ и любых двух симметрий $U$ и $U_{1}$ пуассоновы структуры $L_{U} P$ и $L_{U_{1}} P_{1}$ являются совместимыми по Магри. 
(viii) Любой $C$-инвариантный тензор $N_{\beta \gamma}^{\alpha}$ типа $(1,2)$ и любой $C$-инвариантный тензор $S_{\gamma}^{\alpha \beta}$ типа $(2,1)$ удовлетворяют уравнениям

$$
\begin{aligned}
& \operatorname{rank}\left\|N_{\beta \tau}^{\alpha} U^{\tau}\right\| \leqslant k, \quad \operatorname{rank}\left\|N_{\beta \gamma}^{\tau} \theta_{\tau}\right\| \leqslant k, \\
& \operatorname{rank}\left\|S_{\tau}^{\alpha \beta} U^{\tau}\right\| \leqslant k, \quad \operatorname{rank}\left\|S_{\beta}^{\alpha \tau} \theta_{\tau}\right\| \leqslant k,
\end{aligned}
$$

где $U$ - произвольная симметрия и $\theta$ - произвольная $C$-инвариантная 1-форма. Любые $C$-инвариантные тензор $T_{\alpha \beta \gamma}$ типа $(0,3)$ и тензор $R^{\alpha \beta \gamma}$ типа $(3,0)$ удовлетворяют уравнениям

$$
\operatorname{rank}\left\|T_{\alpha \beta \tau} U^{\tau}\right\| \leqslant k, \quad \operatorname{rank}\left\|R^{\alpha \beta \tau} \theta_{\tau}\right\| \leqslant k .
$$

Любые свертки этих тензоров с двумя произвольными симметриями $U$ и $U_{1}$ или с $C$-инвариантными 1-формами $\theta$ и $\theta_{1}$ равны нулю:

$$
\begin{aligned}
& N_{\tau \nu}^{\alpha} U^{\tau} U_{1}^{\nu}=0, \quad N_{\beta \nu}^{\tau} U^{\nu} \theta_{\tau}=0, \quad S_{\nu}^{\alpha \tau} U^{\nu} \theta_{\tau}=0, \\
& S_{\alpha}^{\tau \nu} \theta_{\tau} \theta_{1 . \nu}=0, \quad T_{\alpha \tau \nu} U^{\tau} U_{1}^{\nu}=0, \quad R^{\alpha \tau \nu} \theta_{\tau} \theta_{1 . \nu}=0 .
\end{aligned}
$$

(ix) Для произвольного $C$-инвариантного тензора $N_{\beta \gamma}^{\alpha}$ типа $(1,2)$ многочлен

$$
P_{N}(u, \lambda)=\operatorname{det}\left(N_{u}-\lambda\right)
$$

приводим и является произведением двух многочленов степени $k$. Здесь $\left(N_{u}\right)_{\gamma}^{\alpha}=$ $N_{\tau \gamma}^{\alpha} u^{\tau}, u \in T\left(M^{2 k}\right)$. Многочлен $P_{N}(u, \lambda)$ удовлетворяет уравнениям

$$
P_{N}(u+U, \lambda)=P_{N}(U, \lambda), \quad P_{N}(U, \lambda)=\lambda^{2 k},
$$

где $U-$ произвольная симметрия.

$(\mathrm{x})$ Для любого $C$-инвариантного тензора $S_{\gamma}^{\alpha \beta}$ типа $(2,1)$ многочлен

$$
P_{S}(\eta, \lambda)=\operatorname{det}\left(S_{\eta}-\lambda\right)
$$

приводим и является произведением двух многочленов степени $k$. Здесь $\left(S_{\eta}\right)_{\gamma}^{\alpha}=$ $S_{\gamma}^{\alpha \tau} \eta_{\tau}, \eta \in T^{*}\left(M^{2 k}\right)$. Уравнения

$$
P_{S}(\eta+\theta, \lambda)=P_{S}(\eta, \lambda), \quad P_{S}(\theta, \lambda)=\lambda^{2 k}
$$

справедливы для любой $C$-инвариантной 1-формы $\theta$.

(xi) Определены кольца $A-B-C$-когомологий

$$
H_{A}^{*}\left(V, M^{n}\right), \quad H_{B}^{*}\left(V, M^{n}\right), \quad H_{C}^{*}\left(V, M^{n}\right)
$$

для произвольной динамической системы на многообразии $M^{n}$. В $\S 13$, используя вьведенные канонические формы для $C$-инвариантных тензоров, доказана бесконечномерность групп когомологий

$$
H_{A}^{2}(V, \mathscr{O}), \quad H_{B}^{2}(V, \mathscr{O}), \quad H_{C}^{2}(V, \mathscr{O})
$$

для $C$-интегрируемых и невырожденных по Пуанкаре гамильтоновых систем в тороидальных областях $\mathscr{O}=B_{a} \times \mathbb{T}^{k}$.

(хіi) В $\S 14$ установлены связи между $B$-когомологиями динамических систем $H_{B}^{*}\left(V, M^{n}\right), B$-когомологиями гладких отображений $H_{B}^{*}\left(f, M^{n}\right)$ и классическими когомологиями де Рама $H^{*}\left(M^{n}\right)$. В теореме 2 доказано, что бесконечномерность групп когомологий $H_{B}^{2}\left(V, M^{2 k}\right)$ и $H_{B}^{4}\left(V, M^{2 k}\right)$ является необходимым условием для невырожденной $C$-интегрируемости динамической системы $V$ на многообразии $M^{2 k}$. 


\section{Список литературы}

1. Bogoyavlenskij O. I. Invariant incompatible Poisson structures // Proc. Roy. Soc. London Ser. A. 1995. V. 450. P. 723-730.

2. Bogoyavlenskij O.I. A cohomology of dynamical systems // Compt. Rend. Mathem. Canada. 1995. V. 17. P. 253-258.

3. Bogoyavlenskij O.I. Theory of tensor invariants of integrable Hamiltonian systems. I. Incompatible Poisson structures // Comm. Math. Phys. 180. № 3. P. 529-586.

4. Bogoyavlenskij O.I. The $A-B$ - $C$-cohomologies for dynamical systems // Compt. Rend. Mathem. Canada. 1996. V. 18. P. 199-204.

5. Bogoyavlenskij O.I. Theory of tensor invariants of integrable Hamiltonian systems. II. Theorem on symmetries and its applications // Comm. Math. Phys. 1997. V. 184. P. 301-365.

6. Арнольд В. И. Математические методы классической механики. М.: Наука, 1974.

7. Liouville J. Notesur l'integration des equations differentielles de la Dynamique // J. Math. Pures Appl. 1855. V. 20. P. 137-138.

8. Арнольд В.И. Малые знаменатели II: Доказательство теоремы А.Н. Колмогорова о сохранении условно-периодических движений при малом изменении функции Гамиль тона // УМН. 1963. Т. 18. № 5. С. 13-40.

9. Колмогоров А.Н. О сохранении условно-периодических движений при малом изменении функции Гамильтона // Докл. АН СССР 1954. V. 98. № 4. Р. 527-530.

10. Колмогоров A.H. Общая теория динамических систем и классическая механика // Proc. Intern. Congr. Math. 1954. T. 1. Amsterdam: North-Holland, 1957. C. 315-333.

11. Moser J. On invariant curves of area-preserving mappings of an annulus // Nachr. Akad. Wiss. Göttingen Math.-Phys. 1962. V. 1. P. 1-20.

12. Moser J. Convergent series expansions for quasi-periodic motions // Math. Ann. 1967. V. 169. P. 137-176.

13. Poincare H. Les metodes nouvelles de la mechanique celeste. V. 1. Paris: Gauthier-Villars, 1892.

14. Nijenhuis A. $X_{n-1}$-forming sets of eigenvectors // Proc. Konink. Nederl. Akad. Wetensch. 1951. V. 54. P. $200-212$.

15. Kobayashi S., Nomizu K. Foundations of differential geometry. New York: Interscience Publishers, 1963.

16. Abraham R., Marsden J. E. Foundations of Mechanics. London: TheBenjamin/Cummings Publishing, 1978.

17. Kozlov $V . V$. Symmetries, topology and resonances in Hamiltonian mechanics. Berlin: Springer-Verlag, 1995.

18. Marsden J. E., Ratiu T. S. Introduction to mechanics and symmetry. Berlin: Springer-Verlag, 1994.

19. Magri F. A simple model of an integrable Hamiltonian system // J. Math. Phys. 1978. V. 19. P. $1156-1162$.

20. De Rham G. Sur l'Analysis sutus des varietes a $n$ dimensions // J. de Mathematiques. 1931. V. 10. P. 115-200.

21. Birkhoff G. D. Dynamical systems. Providence, Rhode Island: Amer. Math. Soc., 1966.

22. Katok A., Hasselblatt B. Introduction to the modern theory of dynamical systems. Cambridge: Cambridge Univ. Press, 1995. 